MATHEMATICS OF COMPUTATION

Volume 73 , Number 245 , Pages 95-125

S $0025-5718(03) 01521-7$

Article electronically published on June 19, 2003

\title{
MIXED LAGUERRE-LEGENDRE PSEUDOSPECTRAL METHOD FOR INCOMPRESSIBLE FLUID FLOW IN AN INFINITE STRIP
}

\author{
BEN-YU GUO AND CHENG-LONG XU
}

\begin{abstract}
In this paper, we investigate the mixed Laguerre-Legendre interpolation approximation and its application. Some approximation results are established. A mixed Laguerre-Legendre pseudospectral scheme is constructed for incompressible fluid flow in an infinite strip. Its stability and convergence are proved. Numerical results show the efficiency of this new approach.
\end{abstract}

\section{INTRODUCTION}

Recently, more and more attention is being paid to fluid flows in unbounded domains and their numerical simulations: see, e.g., Guo and $\mathrm{Xu}$ [13], Kweon and Kellogg [14, and Maday, Pernaud-Thomas and Vandeven [17]. In actual computations, we have to consider three things. The first one is the suitable choice of alternative formulations of partial differential equations governing the movements of fluid flows, which makes calculations simpler. As we know, the Navier-Stokes equation plays an important role in studying incompressible fluid flows. Usually we consider the primitive equation which is quite preferable in theoretical analysis; see, e.g., Lions [15] and Témam [20]. But it is not easy to deal with the incompressibility in computations by using the finite element method or the spectral method. At the same time it is difficult to evaluate the pressure on the boundary, if we use the finite difference method. So it is natural to consider other forms of the Navier-Stokes equation. For instance, we may consider the vorticity-stream function form. In this case, the incompressibility is included automatically, and the pressure no longer appears. However, there is no physical boundary condition on the vorticity. Some authors considered the stream function form of the NavierStokes equation, which avoids all trouble mentioned in the above; see, e.g., Guo and $\mathrm{He}$ [10]. This form might be one of reasonable formulations for numerical simulations of incompressible fluid flows. The second problem is the choice of numerical methods for unbounded domains. The simplest way is to restrict calculations to certain bounded domains and impose some artificial boundary conditions. However, this treatment also causes additional errors. So it seems better to discretize the

Received by the editor August 16, 2001 and, in revised form, April 12, 2002.

2000 Mathematics Subject Classification. Primary 65M70, 41A30, 35Q30.

Key words and phrases. Incompressible fluid flow, infinite strip, Laguerre-Legendre pseudospectral method.

The work of the first author is partially supported by The Special Founds for Major State Basic Research Projects of China G1999032804 and The Shanghai Natural Science Foundation N.00JC14057. 
original problems in unbounded domains directly. For instance, Boyd [5], Coulaud, Funaro and Kavian [6], Funaro and Kavian 8], Guo [9], Guo and Shen [11, Guo and $\mathrm{Xu}$ [12], Maday, Pernaud-Thomas and Vandeven [17], and $\mathrm{Xu}$ and Guo [21] provided various spectral methods for one-dimensional problems. But there are very few works for multiple-dimensional problems. Indeed, the reasonable choice of the spectral method depends on domains. For example, we can use the mixed Laguerre-Legendre spectral method for fluid flows in an infinite strip; see, e.g., Xu and Guo 22. A more preferable method is the mixed Laguerre-Legendre pseudospectral method, since it only needs to evaluate the values of unknown functions on the interpolation points, avoids quadratures over unbounded domains, and can deal with nonlinear terms easily in calculations. Furthermore, for the same domain, the choice of interpolation approximations depends on the differential equations considered, since different equations correspond to different consistent boundary conditions which in turn correspond to different interpolations. The third problem is how to estimate numerical errors. As usual, we first compare numerical solutions with certain orthogonal projections of genuine solutions. For the stream function form, we have to study some projections related to partial differential equations of fourth order. However, there has been only one excellent result, in Bernardi and Maday 4, on the Legendre pseudospectral approximation to fourth-order problems in bounded domains. So far, there is no work concerning the corresponding approximation in unbounded domains.

This paper is devoted to the mixed Laguerre-Legendre pseudospectral method for fluid flow in an infinite strip. Let $\Omega=\{(x, y)|0<x<\infty| y \mid,<1\}$ with the fixed, nonslip wall $\partial \Omega$. Assume $T>0$ and let $\mu$ be the kinetic viscosity. $W(x, y, t)$ and $W_{0}(x, y)$ are the stream function and its initial state, respectively. $F(x, y, t)$ is the source term. For the sake of simplicity, let

$$
G(u, v)=\partial_{y} u \partial_{x} \Delta v-\partial_{x} u \partial_{y} \Delta v .
$$

The stream function form of the Navier-Stokes equation in an infinite strip takes the following form,

$$
\begin{cases}\partial_{t} \triangle W+G(W, W)-\mu \triangle^{2} W=F, & \text { in } \Omega \times(0, T], \\ W=\frac{\partial W}{\partial n}=0, & \text { on } \partial \Omega \times[0, T], \\ W(x, y, 0)=W_{0}(x, y), & \text { in } \Omega .\end{cases}
$$

Besides, the stream function $W$ satisfies certain asymptotic boundary conditions as $x \rightarrow \infty$. For simplicity, we assume that

$$
\lim _{x \rightarrow \infty} W=\lim _{x \rightarrow \infty} \partial_{x} W=0, \quad|y| \leq 1, t \in(0, T] .
$$

We shall construct a scheme for (1.1)-(1.2) by using the Laguerre interpolation approximation in the $x$-direction and the Legendre interpolation approximation in the $y$-direction, give some numerical results, and analyze the numerical errors.

This paper is organized as follows. In the next section, we first recall and establish some results on the Laguerre interpolation and the Legendre interpolation in one dimension, corresponding to pseudospectral methods for fourth-order problems. Then we investigate the mixed Laguerre-Legendre interpolation approximation, which plays an important role in numerical analysis of the related method. In Section 3, we first construct the mixed Laguerre-Legendre pseudospectral scheme 
for solving problem (1.1)-(1.2). Then we give the results on its stability and convergence and present some numerical results showing the efficiency of this new approach. In Section 4, we prove the error estimates. The final section is for some concluding remarks. The main idea and techniques used in this paper are also applicable to other nonlinear problems in multiple-dimensional unbounded domains.

\section{The MiXed LAgUERRE-LEgEndRE INTERPolation APPROXIMATION}

This section is for the mixed Laguerre-Legendre interpolation approximation in two dimensions.

We first consider the Laguerre interpolation. Let $\Lambda_{1}=\{x \mid 0<x<\infty\}$ and let $\chi(x)$ be a certain weight function. For $1 \leq p \leq \infty$, let

$$
L_{\chi}^{p}\left(\Lambda_{1}\right)=\left\{v \mid v \text { is measurable on } \Lambda_{1} \text { and }\|v\|_{L_{\chi}^{p}\left(\Lambda_{1}\right)}<\infty\right\},
$$

where

$$
\|v\|_{L_{\chi}^{p}\left(\Lambda_{1}\right)}= \begin{cases}\left(\int_{\Lambda_{1}}|v(x)|^{p} \chi(x) d x\right)^{\frac{1}{p}}, & 1 \leq p<\infty, \\ \operatorname{ess} \sup _{x \in \Lambda_{1}}|v(x)|, & p=\infty .\end{cases}
$$

The inner product and the norm of the space $L_{\chi}^{2}\left(\Lambda_{1}\right)$ are given by

$$
(u, v)_{\chi, \Lambda_{1}}=\int_{\Lambda_{1}} u(x) v(x) \chi(x) d x, \quad\|v\|_{\chi, \Lambda_{1}}=(v, v)_{\chi, \Lambda_{1}}^{\frac{1}{2}} .
$$

For simplicity, let $\partial_{x} v(x)=\frac{\partial v}{\partial x}(x)$, etc. For any nonnegative integer $m$,

$$
H_{\chi}^{m}\left(\Lambda_{1}\right)=\left\{v \mid \partial_{x}^{k} v \in L_{\chi}^{2}\left(\Lambda_{1}\right), 0 \leq k \leq m\right\}
$$

equipped with the following inner product, seminorm and norm ,

$$
\begin{gathered}
(u, v)_{m, \chi, \Lambda_{1}}=\sum_{k=0}^{m}\left(\partial_{x}^{k} u, \partial_{x}^{k} v\right)_{\chi, \Lambda_{1}}, \\
|v|_{m, \chi, \Lambda_{1}}=\left\|\partial_{x}^{m} v\right\|_{\chi, \Lambda_{1}}, \quad\|v\|_{m, \chi, \Lambda_{1}}=(v, v)_{m, \chi, \Lambda_{1}}^{\frac{1}{2}} .
\end{gathered}
$$

For any real $r>0$, the space $H_{\chi}^{r}\left(\Lambda_{1}\right)$ and its norm $\|v\|_{r, \chi}$ are defined by space interpolation as in Adams 1]. Furthermore, for any nonnegative integer $m$,

$$
H_{0, \chi}^{m}\left(\Lambda_{1}\right)=\left\{v \mid v \in H_{\chi}^{m}\left(\Lambda_{1}\right), \partial_{x}^{k} v(0)=0,0 \leq k \leq m-1\right\} .
$$

In the following discussions, we shall take $\chi(x)$ as $\omega(x)=e^{-x}, \omega^{q}(x)=\omega(q x)$, $q>0$, and $\omega^{(\alpha)}(x)=x^{\alpha} e^{-x}$, respectively. For $\chi(x) \equiv 1$, we denote $H_{\chi}^{m}\left(\Lambda_{1}\right)$ and $H_{0, \chi}^{m}\left(\Lambda_{1}\right)$ by $H^{m}\left(\Lambda_{1}\right)$ and $H_{0}^{m}\left(\Lambda_{1}\right)$. The inner product, seminorm and norm of $H^{r}\left(\Lambda_{1}\right)$ are denoted by $(u, v)_{m, \Lambda_{1}},|v|_{m, \Lambda_{1}}$ and $\|v\|_{m, \Lambda_{1}}$. In particular, $(u, v)_{\Lambda_{1}}=$ $(v, v)_{0, \Lambda_{1}}$ and $\|v\|_{\Lambda_{1}}=\|v\|_{0, \Lambda_{1}}$.

Assume $\alpha>-1$. The generalized Laguerre polynomial of degree $l$ is defined by

$$
x^{\alpha} e^{-x} \mathcal{L}_{l}^{(\alpha)}(x)=\frac{1}{l !} \partial_{x}^{l}\left(x^{l+\alpha} e^{-x}\right) .
$$

By (5.1.7) and (5.1.14) of Szegö [19], we have that

$$
\begin{gathered}
\mathcal{L}_{l}^{(\alpha)}(0)=\left(\begin{array}{c}
l+\alpha \\
l
\end{array}\right), \\
\partial_{x} \mathcal{L}_{l}^{(\alpha)}(x)=-\mathcal{L}_{l-1}^{(\alpha+1)}(x) .
\end{gathered}
$$


The set of the generalized Laguerre polynomials is the $L_{\omega(\alpha)}^{2}\left(\Lambda_{1}\right)$-orthogonal system, namely,

$$
\int_{\Lambda_{1}} \mathcal{L}_{l}^{(\alpha)}(x) \mathcal{L}_{m}^{(\alpha)}(x) \omega^{(\alpha)}(x) d x=\Gamma(\alpha+1)\left(\begin{array}{c}
l+\alpha \\
l
\end{array}\right) \delta_{l, m}, \quad l, m \geq 0,
$$

where $\delta_{l, m}$ is the Kronecker function. In particular, $\mathcal{L}_{l}^{0}(x)=\mathcal{L}_{l}(x)$ is the usual Laguerre polynomial of degree $l$. By (2.1) and (2.2),

$$
\partial_{x}^{2} \mathcal{L}_{l}(0)=\frac{1}{2} l(l-1) .
$$

By (2.2) and (2.3),

$$
\int_{\Lambda_{1}} \partial_{x}^{2} \mathcal{L}_{l}(x) \partial_{x}^{2} \mathcal{L}_{m}(x) x^{2} e^{-x} d x=l(l-1) \delta_{l, m}, \quad l, m \geq 0 .
$$

For any $v \in L_{\omega}^{2}\left(\Lambda_{1}\right)$,

$$
v(x)=\sum_{l=0}^{\infty} \hat{v}_{l} \mathcal{L}_{l}(x)
$$

with the Laguerre coefficients

$$
\hat{v}_{l}=\int_{\Lambda_{1}} v(x) \mathcal{L}_{l}(x) \omega(x) d x, \quad l=0,1,2, \ldots
$$

Now let $N$ be any positive integer and let $\mathcal{P}_{N}\left(\Lambda_{1}\right)$ be the set of restrictions to $\Lambda_{1}$ of all algebraic polynomials of degree at most $N$. Furthermore, $\mathcal{P}_{N}^{0}\left(\Lambda_{1}\right)=$ $\mathcal{P}_{N}^{1,0}\left(\Lambda_{1}\right)=\mathcal{P}_{N}\left(\Lambda_{1}\right) \cap H_{0}^{1}\left(\Lambda_{1}\right)$ and $\mathcal{P}_{N}^{2,0}\left(\Lambda_{1}\right)=\mathcal{P}_{N}\left(\Lambda_{1}\right) \cap H_{0}^{2}\left(\Lambda_{1}\right)$. We denote by $c$ or $c_{i}$ certain generic positive constants independent of any function and $N$.

Lemma 2.1 (see Maday, Pernaud-Thomas and Vandeven [17]). For any $\phi \in$ $\mathcal{P}_{N}\left(\Lambda_{1}\right)$ and $r \geq 0$

$$
\|\phi\|_{r, \omega, \Lambda_{1}} \leq c N^{r}\|\phi\|_{\omega, \Lambda_{1}} .
$$

The $L_{\omega}^{2}\left(\Lambda_{1}\right)$-orthogonal projection $P_{N}: L_{\omega}^{2}\left(\Lambda_{1}\right) \rightarrow \mathcal{P}_{N}\left(\Lambda_{1}\right)$ is a mapping such that for any $v \in L_{\omega}^{2}\left(\Lambda_{1}\right)$,

$$
\left(P_{N} v-v, \phi\right)_{\omega, \Lambda_{1}}=0, \quad \forall \phi \in \mathcal{P}_{N}\left(\Lambda_{1}\right) .
$$

For technical reasons, Bernardi and Maday [4 introduced the space

$$
H_{\omega, \beta}^{r}\left(\Lambda_{1}\right)=\left\{v \mid v, x^{\frac{\beta}{2}} v \in H_{\omega}^{r}\left(\Lambda_{1}\right)\right\},
$$

equipped with the norm $\|v\|_{r, \omega, \beta, \Lambda_{1}}=\left\|v(1+x)^{\frac{\beta}{2}}\right\|_{r, \omega, \Lambda_{1}}$ where $\beta$ is any nonnegative integer.

Lemma 2.2 (see Bernardi and Maday [4]). For any $v \in H_{\omega, \beta}^{r}\left(\Lambda_{1}\right), r \geq 0$ and $0 \leq \mu \leq r$

$$
\left\|P_{N} v-v\right\|_{\mu, \omega, \Lambda_{1}} \leq c N^{\mu-\frac{r}{2}}\|v\|_{r, \omega, \beta, \Lambda_{1}},
$$

where $\beta$ is the largest integer for which $\beta<r+1$.

We next turn to a Gauss-quadrature which induces a suitable interpolation for fourth-order problems. Let $\sigma_{j}^{N}$ be the zeros of the polynomial $\partial_{x}^{2} \mathcal{L}_{N+1}(x)$. By (2.2), they are also the zeros of $\mathcal{L}_{N-1}^{(2)}(x)$. Let $\tilde{\omega}_{j}^{N}$ be the corresponding Christoffel numbers. According to (2.2) and to (3.6.6) of Davis and Rabinowitz [7,

$$
\tilde{\omega}_{j}^{N}=\frac{N(N+1) \sigma_{j}^{N}}{\left(\partial_{x}^{2} \mathcal{L}_{N+2}\left(\sigma_{j}^{N}\right)\right)^{2}} .
$$


Moreover, by (3.6.5) of Davis and Rabinowitz [7], for any $\phi \in \mathcal{P}_{2 N-3}\left(\Lambda_{1}\right)$,

$$
\int_{\Lambda_{1}} \phi(x) x^{2} e^{-x} d x=\sum_{j=1}^{N-1} \phi\left(\sigma_{j}^{N}\right) \tilde{\omega}_{j}^{N} .
$$

Set

$$
\omega_{j}^{N}=\frac{\tilde{\omega}_{j}^{N}}{\left(\sigma_{j}^{N}\right)^{2}}, \quad 1 \leq j \leq N-1 .
$$

In addition, we take $X(x)=\prod_{j=1}^{N-1}\left(x-\sigma_{j}^{N}\right)$ and choose the weights $\xi_{0}^{N}$ and $\xi_{1}^{N}$ in such a way that

$$
\begin{gathered}
\int_{\Lambda_{1}} X(x) x e^{-x} d x=(-1)^{N-1} \xi_{1}^{N} \prod_{j=1}^{N-1} \sigma_{j}^{N}, \\
\int_{\Lambda_{1}} X(x) e^{-x} d x=(-1)^{N-1} \xi_{0}^{N} \prod_{j=1}^{N-1} \sigma_{j}^{N}+(-1)^{N-2} \xi_{1}^{N} \sum_{j=1}^{N-1} \frac{1}{\sigma_{j}^{N}}\left(\prod_{k=1}^{N-1} \sigma_{k}^{N}\right) .
\end{gathered}
$$

Then a calculation shows that (see Appendix A of this paper),

$$
\xi_{1}^{N}=\frac{2}{N(N+1)}, \quad \xi_{0}^{N}=\frac{2(4 N-1)}{3 N(N+1)} .
$$

Lemma 2.3. For any $\phi \in \mathcal{P}_{2 N-1}\left(\Lambda_{1}\right)$ and $N \geq 2$,

$$
\int_{\Lambda_{1}} \phi(x) e^{-x} d x=\sum_{j=1}^{N-1} \phi\left(\sigma_{j}^{N}\right) \omega_{j}^{N}+\phi(0) \xi_{0}^{N}+\partial_{x} \phi(0) \xi_{1}^{N} .
$$

Proof. For any $\phi \in \mathcal{P}_{2 N-1}\left(\Lambda_{1}\right)$, we can rewrite it as

$$
\phi(x)=x^{2} \psi(x)+\eta(x) X(x),
$$

where $\psi \in \mathcal{P}_{2 N-3}\left(\Lambda_{1}\right)$ and $\eta \in \mathcal{P}_{1}\left(\Lambda_{1}\right)$. So it suffices to prove that (2.8) is valid for $\phi_{1}(x)=x^{2} \psi(x), \phi_{2}(x)=x X(x)$ and $\phi_{3}(x)=X(x)$. By virtue of (2.6), (2.8) is true for $\phi_{1}(x)$. Next, $\phi_{2}(0)=\phi_{2}\left(\sigma_{j}^{N}\right)=0,1 \leq j \leq N-1$ and $\partial_{x} \phi_{2}(0)=X(0)$. Thus by the definition of $\xi_{1}^{N},(2.8)$ is also true for $\phi_{2}(x)$. Similarly, a direct calculation shows that (2.8) is valid for $\phi_{3}(x)$. This completes the proof.

We now introduce the discrete inner product and the discrete norm as follows,

$$
(u, v)_{\omega, N, \Lambda_{1}}=\sum_{j=1}^{N-1} u\left(\sigma_{j}^{N}\right) v\left(\sigma_{j}^{N}\right) \omega_{j}^{N}, \quad\|v\|_{\omega, N, \Lambda_{1}}=(v, v)_{\omega, N, \Lambda_{1}}^{\frac{1}{2}} .
$$

By (2.8), for any $\phi \psi \in \mathcal{P}_{2 N-1}^{2,0}\left(\Lambda_{1}\right)$,

$$
(\phi, \psi)_{\omega, N, \Lambda_{1}}=(\phi, \psi)_{\omega, \Lambda_{1}} .
$$

In particular, for any $\phi \in \mathcal{P}_{N-1}^{0}\left(\Lambda_{1}\right)$,

$$
\|\phi\|_{\omega, N, \Lambda_{1}}=\|\phi\|_{\omega, \Lambda_{1}}
$$

Lemma 2.4. For any $\phi \in \mathcal{P}_{N}^{2,0}\left(\Lambda_{1}\right)$,

$$
\frac{1}{\sqrt{N}}\|\phi\|_{\omega, \Lambda_{1}} \leq\|\phi\|_{\omega, N, \Lambda_{1}} \leq\|\phi\|_{\omega, \Lambda_{1}} .
$$


Proof. By (2.5), the set $\left\{x \partial_{x}^{2} \mathcal{L}_{l+1}(x) \mid l=1,2, \cdots, N\right\}$ forms an orthogonal basis of $\mathcal{P}_{N}^{0}\left(\Lambda_{1}\right)$ with the weight $\omega(x)=e^{-x}$. So for any $\phi \in \mathcal{P}_{N}^{0}\left(\Lambda_{1}\right)$,

$$
\phi(x)=\sum_{l=1}^{N} C_{l}^{N} x \partial_{x}^{2} \mathcal{L}_{l+1}(x) .
$$

Consequently we obtain from (2.5) that

$$
\|\phi\|_{\omega, \Lambda_{1}}^{2}=\sum_{l=1}^{N}\left(C_{l}^{N}\right)^{2} \int_{\Lambda_{1}}\left(\partial_{x}^{2} \mathcal{L}_{l+1}(x)\right)^{2} x^{2} e^{-x} d x=\sum_{l=1}^{N} l(l+1)\left(C_{l}^{N}\right)^{2} .
$$

Due to $\partial_{x}^{2} L_{N+1}\left(\sigma_{j}^{N}\right)=0$, we have from (2.5) and (2.9) that

$$
\begin{aligned}
\|\phi\|_{\omega, N, \Lambda_{1}}^{2} & =\sum_{m, l=1}^{N-1} \int_{\Lambda_{1}} C_{m}^{N} C_{l}^{N} \partial_{x}^{2} \mathcal{L}_{m+1}(x) \partial_{x}^{2} \mathcal{L}_{l+1}(x) x^{2} e^{-x} d x \\
& =\sum_{l=1}^{N-1} l(l+1)\left(C_{l}^{N}\right)^{2} .
\end{aligned}
$$

If $\phi \in \mathcal{P}_{N}^{2,0}\left(\Lambda_{1}\right)$, then we deduce from (2.4) that

$$
\partial_{x} \phi(0)=\sum_{l=1}^{N} C_{l}^{N} \partial_{x}^{2} \mathcal{L}_{l+1}(0)=\frac{1}{2} \sum_{l=1}^{N} l(l+1) C_{l}^{N}=0 .
$$

Hence

$$
C_{N}^{N}=-\sum_{l=1}^{N-1} \frac{l(l+1)}{N(N+1)} C_{l}^{N},
$$

and so by the Cauchy inequality,

$$
\begin{aligned}
N(N+1)\left(C_{N}^{N}\right)^{2} & =N(N+1)\left(\sum_{l=1}^{N-1} \frac{l(l+1)}{N(N+1)} C_{l}^{N}\right)^{2} \\
& \leq(N-1) \sum_{l=1}^{N-1} l(l+1)\left(C_{l}^{N}\right)^{2}
\end{aligned}
$$

The combination of (2.11), (2.12) and (2.14) leads to the desired result.

Remark 2.1. The first inequality in Lemma 2.4 cannot be improved.

Remark 2.2. We can see from (2.11) and (2.12) that for any $\phi \in \mathcal{P}_{N}^{0}\left(\Lambda_{1}\right)$,

$$
\|\phi\|_{\omega, N, \Lambda_{1}} \leq\|\phi\|_{\omega, \Lambda_{1}} .
$$

Remark 2.3. We also have that for any $\phi \in \mathcal{P}_{N-1}\left(\Lambda_{1}\right)$,

$$
\|\phi\|_{\omega, N, \Lambda_{1}} \leq c\|\phi\|_{\omega, \Lambda_{1}} .
$$

Indeed, (2.8) implies

$$
\int_{\Lambda_{1}} \phi^{2}(x) e^{-x} d x=\sum_{j=1}^{N-1} \phi^{2}\left(\sigma_{j}^{N}\right) \omega_{j}^{N}+\phi^{2}(0) \xi_{0}^{N}+2 \phi(0) \partial_{x} \phi(0) \xi_{1}^{N} .
$$

By the Trace Theorem (see, e.g., Theorem 3.2 of Lions and Magenes [16]),

$$
|\phi(0)|=\left|\left(e^{-\frac{x}{2}} \phi\right)(0)\right| \leq c\left\|e^{-\frac{x}{2}} \phi\right\|_{H^{\frac{1}{2}}\left(\Lambda_{1}\right)} .
$$


According to Proposition 3.1 of Maday, Pernaud-Thomas and Vandeven [17], the mapping $v \rightarrow e^{-\frac{x}{2}} v$ is an isomorphism from $H^{r}\left(\Lambda_{1}\right)$ to $H_{\omega}^{r}\left(\Lambda_{1}\right)$. So

$$
\begin{gathered}
|\phi(0)| \leq c\|\phi\|_{H_{\omega}^{\frac{1}{2}\left(\Lambda_{1}\right)}} \leq c N^{\frac{1}{2}}\|\phi\|_{\omega, \Lambda_{1}}, \\
\left|\partial_{x} \phi(0)\right| \leq c\left\|e^{-\frac{x}{2}} \partial_{x} \phi\right\|_{H^{\frac{1}{2}\left(\Lambda_{1}\right)}} \leq c\|\phi\|_{H_{\omega}^{\frac{3}{2}}\left(\Lambda_{1}\right)} \leq c N^{\frac{3}{2}}\|\phi\|_{\omega, \Lambda_{1}} .
\end{gathered}
$$

The combination of (2.7) and (2.16)-(2.18) leads to the desired result.

We now study the Laguerre interpolation. Let $\Lambda_{N}=\left\{\sigma_{j}^{N} \mid 1 \leq j \leq N-1\right\}$. For any $v \in C^{1}\left(\bar{\Lambda}_{1}\right)$, the interpolant $I_{N} v \in \mathcal{P}_{N}\left(\Lambda_{1}\right)$ such that

$$
\begin{cases}I_{N} v(x)=v(x), & x \in \Lambda_{N} \\ \partial_{x}^{k} I_{N} v(0)=\partial_{x}^{k} v(0), & k=0,1 .\end{cases}
$$

For deriving the approximation accuracy, we follow Mastroianni and Monegato [18 to introduce another space. For $0<q<1$ and $r \geq 0$,

$$
H_{\omega^{q}, *}^{r}\left(\Lambda_{1}\right)=\left\{v \mid v \in L_{\omega^{q}}^{2}\left(\Lambda_{1}\right),\|v\|_{H_{\omega q, *}^{r}\left(\Lambda_{1}\right)}<\infty\right\},
$$

where

$$
\|v\|_{H_{\omega q, *}^{r}\left(\Lambda_{1}\right)}=\left(\sum_{l=0}^{\infty}(l+1)^{r} \hat{\hat{v}}_{l}^{2}\right)^{\frac{1}{2}}
$$

and $\hat{\hat{v}}_{l}$ is the coefficients of the expansion of $v$ in terms of the generalized Laguerre polynomials which are mutually orthogonal with the weight $\omega^{q}(x)$. Next, for $r \geq 2$,

$$
\bar{H}_{\omega q}^{r}\left(\Lambda_{1}\right)=\left\{v \mid v \in H_{\omega^{q}}^{2}\left(\Lambda_{1}\right),\|v\|_{\bar{H}_{\omega q}^{r}\left(\Lambda_{1}\right)}<\infty\right\}
$$

where

$$
\|v\|_{\bar{H}_{\omega q}^{r}\left(\Lambda_{1}\right)}=\left\|\partial_{x}^{2} v\right\|_{H_{\omega,}^{r-2}\left(\Lambda_{1}\right)} .
$$

Lemma 2.5. For any $v \in H_{\omega^{q}}^{r}\left(\Lambda_{1}\right), 0<q<1$ and $r \geq 2$,

$$
\left\|v-I_{N} v\right\|_{\omega, \Lambda_{1}} \leq c N^{1-\frac{r}{2}}\|v\|_{r, \omega^{q}, \Lambda_{1}} .
$$

Proof. We first suppose that $v \in H_{0, \omega^{q}}^{2}\left(\Lambda_{1}\right)$. Take $q<q_{1}<1,0<\alpha<1-q_{1}$ and $0<\epsilon<\frac{1}{2}$. The orthogonal projection $P_{N, q_{1}}^{2,0}: H_{0, \omega^{q_{1}}}^{2}\left(\Lambda_{1}\right) \rightarrow \mathcal{P}_{N}^{2,0}\left(\Lambda_{1}\right)$ is a mapping such that for any $v \in H_{0, \omega^{q_{1}}}^{2}\left(\Lambda_{1}\right)$,

$$
\int_{\Lambda_{1}} \partial_{x}^{2}\left(v-P_{N, q_{1}}^{2,0} v\right) \partial_{x}^{2} \phi \omega^{q_{1}}(x) d x=0, \quad \forall \phi \in \mathcal{P}_{N}^{2,0}\left(\Lambda_{1}\right) .
$$

By Theorem 2.1 of Xu and Guo [22], we have that for any $v \in \bar{H}_{\omega^{q_{1}}}^{r}\left(\Lambda_{1}\right) \cap H_{0, \omega^{q_{1}}}^{2}\left(\Lambda_{1}\right)$,

$$
\left\|v-P_{N, q_{1}}^{2,0} v\right\|_{\omega, \Lambda_{1}} \leq c_{1} N^{-\frac{r}{2}}\|v\|_{\bar{H}_{\omega}^{r} q_{1}\left(\Lambda_{1}\right)} .
$$

Furthermore, it is shown in Appendix B of this paper that for any $v \in H_{\omega^{1-\alpha}}^{\frac{1}{2}+\epsilon}\left(\Lambda_{1}\right)$, $0<\alpha<1$ and $0<\epsilon<1$,

$$
\|v\|_{\omega, N, \Lambda_{1}}=\left\|I_{N} v\right\|_{\omega, N, \Lambda_{1}} \leq c\|v\|_{H_{\omega^{1-\alpha}}^{\frac{1}{2}+\epsilon}\left(\Lambda_{1}\right)}^{\frac{1}{2}}\|v\|_{H_{\omega^{1-\alpha}}^{\frac{1}{2}-\epsilon}\left(\Lambda_{1}\right)}^{\frac{1}{2}} .
$$


Since $I_{N} v-P_{N, q_{1}}^{2,0} v \in \mathcal{P}_{N}^{2,0}\left(\Lambda_{1}\right)$, we have from Lemma 2.4 that

$$
\begin{aligned}
\left\|I_{N} v-P_{N, q_{1}}^{2,0} v\right\|_{\omega, \Lambda_{1}} & \leq c N^{\frac{1}{2}}\left\|I_{N}\left(v-P_{N, q_{1}}^{2,0} v\right)\right\|_{\omega, N, \Lambda_{1}} \\
& \leq c N^{\frac{1}{2}}\left\|v-P_{N, q_{1}}^{2,0} v\right\|_{H_{\omega^{1-\alpha}}^{\frac{1}{2}+\epsilon}\left(\Lambda_{1}\right)}^{\frac{1}{2}}\left\|v-P_{N, q_{1}}^{2,0} v\right\|_{H_{\omega^{1-\alpha}}^{\frac{1}{2}-\epsilon}\left(\Lambda_{1}\right)}^{\frac{1}{2}} \\
& \leq c N^{1-\frac{r}{2}}\|v\|_{\bar{H}_{\omega}^{r} q_{1}\left(\Lambda_{1}\right)} .
\end{aligned}
$$

Thus we deduce from (2.19) and (2.21) that

$$
\begin{aligned}
\left\|v-I_{N} v\right\|_{\omega, \Lambda_{1}} & \leq\left\|v-P_{N, q_{1}}^{2,0} v\right\|_{\omega, \Lambda_{1}}+\left\|I_{N} v-P_{N, q_{1}}^{2,0} v\right\|_{\omega, \Lambda_{1}} \\
& \leq c N^{1-\frac{r}{2}}\|v\|_{\bar{H}_{\omega}^{r} q_{1}\left(\Lambda_{1}\right)} \leq c N^{1-\frac{r}{2}}\|v\|_{r, \omega^{q}, \Lambda_{1}} .
\end{aligned}
$$

Next, for any $v \in H_{\omega^{q}}^{r}\left(\Lambda_{1}\right)$, we take $v^{*} \in \mathcal{P}_{1}\left(\Lambda_{1}\right)$ such that $v-v^{*} \in H_{0, \omega^{q}}^{2}\left(\Lambda_{1}\right)$. Then by the definition of the space $\bar{H}_{\omega^{q_{1}}}^{r}\left(\Lambda_{1}\right)$ and Lemma 2.3 of Mastroianni and Monegato [18,

$$
\left\|v-v^{*}\right\|_{\bar{H}_{\omega}^{r} q_{1}\left(\Lambda_{1}\right)}=\left\|\partial_{x}^{2} v\right\|_{H_{\omega q_{1}, *}^{r-2}\left(\Lambda_{1}\right)} \leq c\left\|\partial_{x}^{2} v\right\|_{r-2, \omega^{q}, \Lambda_{1}} \leq c\|v\|_{r, \omega^{q}, \Lambda_{1}} .
$$

This fact with (2.22) implies that

$$
\left\|v-I_{N} v\right\|=\left\|v-v^{*}-I_{N}\left(v-v^{*}\right)\right\| \leq c N^{1-\frac{r}{2}}\left\|v-v^{*}\right\|_{\bar{H}_{\omega}^{r} q_{1}\left(\Lambda_{1}\right)} \leq c N^{1-\frac{r}{2}}\|v\|_{r, \omega^{q}, \Lambda_{1}} .
$$

Theorem 2.1. For any $v \in H_{\omega^{q}}^{r}\left(\Lambda_{1}\right), 0<q<1, r \geq 2$ and $0 \leq \mu \leq r$,

$$
\left\|v-I_{N} v\right\|_{\mu, \omega, \Lambda_{1}} \leq c N^{\mu+1-\frac{r}{2}}\|v\|_{r, \omega^{q}, \Lambda_{1}} .
$$

Proof. By Lemma 2.1,

$$
\left\|P_{N} v-I_{N} v\right\|_{\mu, \omega, \Lambda_{1}} \leq c N^{\mu}\left(\left\|v-P_{N} v\right\|_{\omega, \Lambda_{1}}+\left\|v-I_{N} v\right\|_{\omega, \Lambda_{1}}\right) .
$$

Hence we obtain from Lemmas 2.2 and 2.5 that

$$
\begin{aligned}
\left\|v-I_{N} v\right\|_{\mu, \omega, \Lambda_{1}} & \leq\left\|v-P_{N} v\right\|_{\mu, \omega, \Lambda_{1}}+\left\|P_{N} v-I_{N} v\right\|_{\mu, \omega, \Lambda_{1}} \\
& \leq c N^{\mu-\frac{r}{2}}\|v\|_{r, \omega, \beta, \Lambda_{1}}+c N^{\mu}\left(N^{-\frac{r}{2}}\|v\|_{r, \omega, \beta, \Lambda_{1}}+N^{1-\frac{r}{2}}\|v\|_{r, \omega^{q}, \Lambda_{1}}\right) \\
& \leq c N^{\mu+1-\frac{r}{2}}\|v\|_{r, \omega^{q}, \Lambda_{1}} .
\end{aligned}
$$

We next consider the Legendre interpolation in the $y$-direction. Let $\Lambda_{2}=(-1,1)$. For any $r \geq 0$, we define the spaces $H^{r}\left(\Lambda_{2}\right)$ and $H_{0}^{r}\left(\Lambda_{2}\right)$ as usual. The inner product, the seminorm and the norm of $H^{r}\left(\Lambda_{2}\right)$ are denoted by $(u, v)_{r, \Lambda_{2}},|v|_{r, \Lambda_{2}}$ and $\|v\|_{r, \Lambda_{2}}$. In particular, $(u, v)_{\Lambda_{2}}=(u, v)_{0, \Lambda_{2}}$ and $\|v\|_{\Lambda_{2}}=\|v\|_{0, \Lambda_{2}}$.

Let $L_{l}(y)$ be the Legendre polynomial of degree $l$. The set of the Legendre polynomials is the $L^{2}\left(\Lambda_{2}\right)$-orthogonal system. Moreover, by (3.7) of Bernardi and Maday [3],

$$
\partial_{y}^{2}\left(\left(1-y^{2}\right) \partial_{y}^{2} L_{l}(y)\right)=l(l+2)\left(l^{2}-1\right) L_{l}(y) .
$$

Multiplying the above formula by $L_{m}(y)$ and integrating by parts, we get that

$$
\int_{\Lambda_{2}}\left(1-y^{2}\right)^{2} \partial_{y}^{2} L_{l}(y) \partial_{y}^{2} L_{m}(y) d y=l(l+2)\left(l^{2}-1\right)\left(l+\frac{1}{2}\right)^{-1} \delta_{l, m}, \quad l, m \geq 0 .
$$

For any $v \in L^{2}\left(\Lambda_{2}\right)$,

$$
v(y)=\sum_{l=0}^{\infty} \tilde{v}_{l} L_{l}(y)
$$


with the Legendre coefficients

$$
\tilde{v}_{l}=\left(l+\frac{1}{2}\right) \int_{\Lambda_{2}} v(y) L_{l}(y) d y, \quad l=0,1, \ldots .
$$

Let $M$ be any positive integer and let $\mathcal{P}_{M}\left(\Lambda_{2}\right)$ be the set of restrictions to $\Lambda_{2}$ of all algebraic polynomials of degree at most $M . \mathcal{P}_{M}^{0}\left(\Lambda_{2}\right)=\mathcal{P}_{M}^{1,0}\left(\Lambda_{2}\right)=\mathcal{P}_{M}\left(\Lambda_{2}\right) \cap H_{0}^{1}\left(\Lambda_{2}\right)$ and $\mathcal{P}_{M}^{2,0}\left(\Lambda_{2}\right)=\mathcal{P}_{M}\left(\Lambda_{2}\right) \cap H_{0}^{2}\left(\Lambda_{2}\right)$.

Lemma 2.6 (see Bernardi and Maday [4]). For any $\phi \in \mathcal{P}_{M}\left(\Lambda_{2}\right)$ and $r \geq 0$,

$$
\|\phi\|_{r, \Lambda_{2}} \leq c M^{2 r}\|\phi\|_{\Lambda_{2}} \text {. }
$$

Now we follow the idea of Bernardi and Maday (see page 319 of [4]) to consider an interpolation which is suitable for numerical solutions of fourth-order problems. Let $\tau_{j}^{M}, 1 \leq j \leq M-3$, be the zeros of the polynomial $\partial_{x}^{2} L_{M-1}(y)$, while the $\tilde{\rho}_{j}^{M}$ are the corresponding Christoffel numbers, as in (4.9) of Bernardi and Maday [4], with $m=2$ and $N$ replaced by $N-1$. Let

$$
\rho_{j}^{M}=\left(1-\left(\tau_{j}^{M}\right)^{2}\right)^{-2} \tilde{\rho}_{j}^{M}, \quad 1 \leq j \leq M-3 .
$$

In addition,

$$
\rho_{1,-}^{M}=-\rho_{1,+}^{M}=\frac{8}{M(M-2)\left(M^{2}-1\right)}, \quad \rho_{0,-}^{M}=\rho_{0,+}^{M}=\frac{8\left(2 M^{2}-2 M-1\right)}{3 M(M-2)\left(M^{2}-1\right)} .
$$

By virtue of (4.3) and (4.29) of Bernardi and Maday [4], for any $\phi \in \mathcal{P}_{2 M-3}\left(\Lambda_{2}\right)$,

$$
\int_{\Lambda_{2}} \phi(y) d y=\sum_{j=1}^{M-3} \phi\left(\tau_{j}^{M}\right) \rho_{j}^{M}+\sum_{k=0}^{1}\left(\partial_{y}^{k} \phi(-1) \rho_{k,-}^{M}+\partial_{y}^{k} \phi(1) \rho_{k,+}^{M}\right) .
$$

We next introduce the discrete inner product and the discrete norm as follows,

$$
(u, v)_{M, \Lambda_{2}}=\sum_{j=1}^{M-3} u\left(\tau_{j}^{M}\right) v\left(\tau_{j}^{M}\right) \rho_{j}^{M}, \quad\|v\|_{M, \Lambda_{2}}=(v, v)_{M, \Lambda_{2}}^{\frac{1}{2}}
$$

We get from (2.25) that for any $\phi \psi \in \mathcal{P}_{2 M-3}^{2,0}\left(\Lambda_{2}\right)$,

$$
(\phi, \psi)_{M, \Lambda_{2}}=(\phi, \psi)_{\Lambda_{2}} \text {. }
$$

In particular, for any $\phi \in \mathcal{P}_{M-2}^{0}\left(\Lambda_{2}\right)$,

$$
\|\phi\|_{M, \Lambda_{2}}=\|\phi\|_{\Lambda_{2}} \text {. }
$$

Lemma 2.7 (Bernardi and Maday [4] (16.10) and (16.13)]). For any $\phi, \psi \in$ $\mathcal{P}_{M}^{2,0}\left(\Lambda_{2}\right)$,

$$
\begin{gathered}
\left|(\phi, \psi)_{M, \Lambda_{2}}\right| \leq c\|\phi\|_{\Lambda_{2}}\|\psi\|_{\Lambda_{2}}, \\
c_{1} M^{-1}\|\phi\|_{\Lambda_{2}}^{2} \leq\|\phi\|_{M, \Lambda_{2}}^{2} \leq c_{2}\|\phi\|_{\Lambda_{2}}^{2} .
\end{gathered}
$$

Remark 2.4. It can be verified that for any $\phi \in \mathcal{P}_{M-1}^{0}\left(\Lambda_{2}\right)$,

$$
\|\phi\|_{M, \Lambda_{2}} \leq\|\phi\|_{\Lambda_{2}} \text {. }
$$

Indeed, by (2.23), the set $\left\{\left(1-y^{2}\right) \partial_{y}^{2} L_{l}(y) \mid 2 \leq l \leq M-1\right\}$ is an orthogonal basis of $\mathcal{P}_{M-1}^{0}\left(\Lambda_{2}\right)$, and so for any $\phi \in \mathcal{P}_{M-1}^{0}\left(\Lambda_{2}\right)$,

$$
\phi(y)=\sum_{l=2}^{M-1} \tilde{\tilde{\phi}}_{l}\left(1-y^{2}\right) \partial_{y}^{2} L_{l}(y)
$$


where the $\tilde{\tilde{\phi}}_{l}$ are the corresponding coefficients in the expansions in terms of $\left(1-y^{2}\right) \partial_{y}^{2} L_{l}(y)$. Thus by $(2.26)$ and the fact that $\partial_{y}^{2} L_{M-1}\left(\tau_{j}^{M}\right)=0$, we obtain that

$$
\begin{aligned}
\|\phi\|_{M, \Lambda_{2}}^{2} & =\sum_{l=2}^{M-2}\left(\tilde{\tilde{\phi}}_{l}\right)^{2} \int_{\Lambda_{2}}\left(1-y^{2}\right)^{2}\left(\partial_{y}^{2} L_{l}(y)\right)^{2} d y \\
& \leq \sum_{l=2}^{M-1}\left(\tilde{\tilde{\phi}}_{l}\right)^{2} \int_{\Lambda_{2}}\left(1-y^{2}\right)^{2}\left(\partial_{y}^{2} L_{l}(y)\right)^{2} d y=\|\phi\|_{M, \Lambda_{2}}^{2} .
\end{aligned}
$$

Remark 2.5. We also have that for any $\phi \in \mathcal{P}_{M-2}\left(\Lambda_{2}\right)$,

$$
\|\phi\|_{M, \Lambda_{2}} \leq c\|\phi\|_{\Lambda_{2}} .
$$

In fact, $\phi^{2} \in \mathcal{P}_{2 M-4}\left(\Lambda_{2}\right)$ and so by $(2.25)$,

$$
\begin{aligned}
\int_{\Lambda_{2}} \phi^{2}(y) d y=\sum_{j=1}^{M-3} & \phi\left(\tau_{j}^{M}\right) \rho_{j}^{M}+\phi^{2}(-1) \rho_{0,-}^{M}+\phi^{2}(1) \rho_{0,+}^{M} \\
& +2 \phi(-1) \partial_{x} \phi(-1) \rho_{1,-}^{M}+2 \phi(1) \partial_{x} \phi(1) \rho_{1,+}^{M} .
\end{aligned}
$$

Thanks to the Trace Theorem and Lemma 2.6,

$$
\begin{gathered}
|\phi(0)| \leq c\|\phi\|_{H^{\frac{1}{2}\left(\Lambda_{1}\right)}} \leq c M\|\phi\|_{\Lambda_{2}}, \\
\left|\partial_{x} \phi(0)\right| \leq c\left\|\partial_{x} \phi\right\|_{H^{\frac{1}{2}\left(\Lambda_{1}\right)}} \leq c M^{3}\|\phi\|_{\Lambda_{2}} .
\end{gathered}
$$

Then the conclusion comes from (2.24), (2.28) and the above statements.

Lemma 2.8 (Bernardi, Coppoletta and Maday [2, Lemma 3.2]). For any $\phi \in$ $\mathcal{P}_{M}^{2,0}\left(\Lambda_{2}\right)$,

$$
\left\|\partial_{y} \phi\right\|_{\Lambda_{2}}^{2} \leq-\left(\partial_{y}^{2} \phi, \phi\right)_{M, \Lambda_{2}} \leq c\left\|\partial_{y} \phi\right\|_{\Lambda_{2}}^{2} .
$$

We now study the Legendre interpolation. Let $\Lambda_{M}=\left\{\tau_{j}^{M} \mid 1 \leq j \leq M-3\right\}$. For any $C^{1}\left(\bar{\Lambda}_{2}\right)$, the interpolant $I_{M} v \in \mathcal{P}_{M}\left(\Lambda_{2}\right)$ such that

$$
\begin{cases}I_{M} v(y)=v(y), & y \in \Lambda_{M}, \\ \partial_{y}^{k} I_{M} v( \pm 1)=\partial_{y}^{k} v( \pm 1), & k=0,1 .\end{cases}
$$

Lemma 2.9 (Bernardi and Maday [3, (13.30)]). For any $v \in H^{r}\left(\Lambda_{2}\right), r>\frac{6+\mu}{4}$ and $0 \leq \mu \leq \min (r, 2)$,

$$
\left\|v-I_{M} v\right\|_{\mu, \Lambda_{2}} \leq c M^{\mu-r}\|v\|_{r, \Lambda_{2}} .
$$

Now we are in a position of investigating the mixed Laguerre-Legendre interpolation. Let $\Omega=\Lambda_{1} \times \Lambda_{2}$. For any weight function $\chi(x)$ and $r \geq 0$, the space $H_{\chi}^{r}(\Omega)$ is defined in a similar way as in the previous paragraphs. The seminorm and the norm of $H_{\chi}^{r}(\Omega)$ are denoted by $|v|_{r, \chi}$ and $\|v\|_{r, \chi}$, respectively. For any nonnegative integer $m,(u, v)_{m, \chi}$ stands for the inner product of $H_{\chi}^{m}(\Omega)$. In particular, $(u, v)_{\chi}=(u, v)_{0, \chi}$ and $\|v\|_{\chi}=\|v\|_{0, \chi}$. For $\chi \equiv 1$, we denote $(u, v)_{\chi},|v|_{\chi}$ and $\|v\|_{\chi}$ by $(u, v),|v|$ and $\|v\|$, for simplicity. Furthermore let

$$
\begin{gathered}
H_{0, \omega^{q}}^{1}(\Omega)=\left\{v \mid v \in H_{\omega^{q}}^{1}(\Omega), v(0, y)=v(x,-1)=v(x, 1)=0\right\}, \\
H_{0, \omega^{q}}^{2}(\Omega)=\left\{v \mid v \in H_{\omega^{q}}^{2}(\Omega), v(0, y)=\partial_{x} v(0, y)=v(x,-1)\right. \\
\left.=v(x, 1)=\partial_{y} v(x,-1)=\partial_{y} v(x, 1)=0\right\} .
\end{gathered}
$$


Let $V_{N, M}=\mathcal{P}_{N}\left(\Lambda_{1}\right) \otimes \mathcal{P}_{M}\left(\Lambda_{2}\right)$ and $V_{N, M}^{i, 0}=\mathcal{P}_{N}^{i, 0}\left(\Lambda_{1}\right) \otimes \mathcal{P}_{M}^{i, 0}\left(\Lambda_{2}\right), i=1,2$. The $H_{0, \omega^{q}}^{2}(\Omega)$-orthogonal projection $P_{N, M, q}^{2,0}: H_{0, \omega^{q}}^{2}(\Omega) \rightarrow V_{N, M}^{2,0}$ is a mapping such that for any $H_{0, \omega^{q}}^{2}(\Omega)$,

$$
\left(\Delta\left(v-P_{N, M, q}^{2,0} v\right), \Delta \phi\right)_{\omega^{q}}=0, \quad \forall \phi \in V_{N, M}^{2,0}(\Omega) .
$$

For technical reasons, we introduce a nonisotropic space. For any $0<q \leq 1$ and $r, s \geq 2$, let

$$
\begin{aligned}
M_{q}^{r, s}(\Omega)= & L^{2}\left(\Lambda_{2}, \bar{H}_{\omega^{q}}^{r}\left(\Lambda_{1}\right)\right) \bigcap H^{s}\left(\Lambda_{2}, L_{\omega^{q}}^{2}\left(\Lambda_{1}\right)\right) \bigcap H^{1}\left(\Lambda_{2}, \bar{H}_{\omega^{q}}^{r-1}\left(\Lambda_{1}\right)\right) \\
& \bigcap H^{s-1}\left(\Lambda_{2}, H_{\omega^{q}}^{1}\left(\Lambda_{1}\right)\right) \bigcap H^{2}\left(\Lambda_{2}, \bar{H}_{\omega^{q}}^{r-2}\left(\Lambda_{1}\right)\right) \bigcap H^{s-2}\left(\Lambda_{2}, \bar{H}_{\omega^{q}}^{2}\left(\Lambda_{1}\right)\right),
\end{aligned}
$$

equipped with the norm

$$
\begin{aligned}
\|v\|_{M_{q}^{r}}^{r, s}= & \left(\|v\|_{L^{2}\left(\Lambda_{2}, \bar{H}_{\omega q}^{r}\left(\Lambda_{1}\right)\right)}^{2}+\|v\|_{H^{s}\left(\Lambda_{2}, L_{\omega q}^{2}\left(\Lambda_{1}\right)\right)}^{2}+\|v\|_{H^{1}\left(\Lambda_{2}, \bar{H}_{\omega q}^{r-1}\left(\Lambda_{1}\right)\right)}^{2}\right. \\
& \left.+\|v\|_{H^{s-1}\left(\Lambda_{2}, H_{\omega q}^{1}\left(\Lambda_{1}\right)\right)}^{2}+\|v\|_{H^{2}\left(\Lambda_{2}, \bar{H}_{\omega q}^{r-2}\left(\Lambda_{1}\right)\right)}^{2}+\|v\|_{H^{s-2}\left(\Lambda_{2}, \bar{H}_{\omega q}^{2}\left(\Lambda_{1}\right)\right)}^{2}\right)^{\frac{1}{2}} .
\end{aligned}
$$

Lemma 2.10 (Xu and Guo [22, Remark 2.4]). For any $v \in M_{q}^{r, s}(\Omega) \bigcap H_{0, \omega^{q}}^{2}(\Omega)$, $0<q<1, r, s \geq 2$ and $0 \leq \mu \leq 2$,

$$
\left\|v-P_{N, M, q}^{2,0} v\right\|_{\mu, \omega} \leq c\left(N^{1-\frac{r}{2}}+M^{2-s}\right)\left(N^{-1}+M^{-2}\right)^{1-\frac{\mu}{2}}\|v\|_{M_{q}^{r, s} .}
$$

Next, let $\omega_{i, j}^{N, M}=\omega_{i}^{N} \rho_{j}^{M}$. The discrete inner products are given by

$$
\begin{gathered}
(u, v)_{\omega, N, M}=(u, v)_{0, \omega, N, M}=\sum_{i=1}^{N-1} \sum_{j=1}^{M-3} u\left(\sigma_{i}^{N}, \tau_{j}^{M}\right) v\left(\sigma_{i}^{N}, \tau_{j}^{M}\right) \omega_{i, j}^{N, M}, \\
(u, v)_{1, \omega, N, M}=\sum_{i=1}^{N-1} \sum_{j=1}^{M-3}\left(\partial_{x} u\left(\sigma_{i}^{N}, \tau_{j}^{M}\right) \partial_{x} v\left(\sigma_{i}^{N}, \tau_{j}^{M}\right)\right. \\
\left.+\partial_{y} u\left(\sigma_{i}^{N}, \tau_{j}^{M}\right) \partial_{y} v\left(\sigma_{i}^{N}, \tau_{j}^{M}\right)\right) \omega_{i, j}^{N, M}, \\
(u, v)_{2, \omega, N, M}=\sum_{i=1}^{N-1} \sum_{j=1}^{M-3}\left(\partial_{x}^{2} u\left(\sigma_{i}^{N}, \tau_{j}^{M}\right) \partial_{x}^{2} v\left(\sigma_{i}^{N}, \tau_{j}^{M}\right)\right. \\
+2 \partial_{x} \partial_{y} u\left(\sigma_{i}^{N}, \tau_{j}^{M}\right) \partial_{x} \partial_{y} v\left(\sigma_{i}^{N}, \tau_{j}^{M}\right) \\
+ \\
\left.\partial_{y}^{2} u\left(\sigma_{i}^{N}, \tau_{j}^{M}\right) \partial_{y}^{2} v\left(\sigma_{i}^{N}, \tau_{j}^{M}\right)\right) \omega_{i, j}^{N, M} .
\end{gathered}
$$

Obviously,

$$
\left|(u, v)_{k, \omega, N, M}\right| \leq\|u\|_{k, \omega, N, M}\|v\|_{k, \omega, N, M}, \quad 0 \leq k \leq 2 .
$$

Thanks to Remark 2.2 and Lemma 2.7, for any $\phi, \psi \in P_{N}^{0}\left(\Lambda_{1}\right) \times P_{M}^{2,0}\left(\Lambda_{2}\right)$,

$$
(\phi, \psi)_{\omega, N, M} \leq c\|\phi\|_{\omega}\|\psi\|_{\omega} .
$$

By Lemmas 2.4 and 2.7, for any $\phi \in V_{N, M}^{2,0}$,

$$
c_{1}(M N)^{-\frac{1}{2}}\|\phi\|_{\omega} \leq\|\phi\|_{\omega, N, M} \leq c_{2}\|\phi\|_{\omega} .
$$

Remark 2.6. If $\phi \in V_{N-1, M-2}$, then by Remarks 2.3 and 2.5,

$$
\|\phi\|_{\omega, N, M} \leq c\|\phi\|_{\omega} .
$$


Finally we study the mixed Laguerre-Legendre interpolation. Let $\Omega_{N, M}=\Lambda_{N} \times$ $\Lambda_{M}$. For any $v \in C^{1}(\bar{\Omega})$, the interpolant $I_{N, M} v \in V_{N, M}$ such that

$$
\begin{cases}I_{N, M} v(x, y)=v(x, y), & \forall(x, y) \in \Omega_{N, M}, \\ \partial_{y}^{k} I_{N, M} v(x, \pm 1)=\partial_{y}^{k} v(x, \pm 1), & x \in \Lambda_{1}, k=0,1, \\ \partial_{x}^{k} I_{N, M} v(0, y)=\partial_{x}^{k} v(0, y), & y \in \Lambda_{2}, k=0,1 .\end{cases}
$$

It is easy to see that for any $u, v \in C^{1}(\bar{\Omega})$,

$$
I_{N, M} v=I_{N} I_{M} v=I_{M} I_{N} v, \quad\left(I_{N, M} u-u, v\right)_{\omega, N, M}=0 .
$$

Theorem 2.2. Let $0 \leq \alpha \leq r, 0 \leq \gamma \leq \min (s, 2), r \geq 2, s>\frac{6+\gamma}{4}$ and $0<q<1$. Then for any $v \in H^{\gamma}\left(\Lambda_{2}, H_{\omega^{q}}^{r}\left(\Lambda_{1}\right)\right) \cap H^{s}\left(\Lambda_{2}, H_{\omega^{q}}^{\alpha}\left(\Lambda_{1}\right)\right)$, $\left\|v-I_{N, M} v\right\|_{H^{\gamma}\left(\Lambda_{2}, H_{\omega}^{\alpha}\left(\Lambda_{1}\right)\right)} \leq c N^{\alpha+1-\frac{r}{2}}\|v\|_{H^{\gamma}\left(\Lambda_{2}, H_{\omega}^{r} q\left(\Lambda_{1}\right)\right)}+c M^{\gamma-s}\|v\|_{H^{s}\left(\Lambda_{2}, H_{\omega q}^{\alpha}\left(\Lambda_{1}\right)\right)}$.

Proof. Let $\mathcal{I}$ be the identity operator. Then

$$
v-I_{N, M} v=\left(v-I_{N} v\right)+\left(v-I_{M} v\right)-\left(\mathcal{I}-I_{N}\right)\left(\mathcal{I}-I_{M}\right) v .
$$

Hence

$$
\left\|v-I_{N, M} v\right\|_{H^{\gamma}\left(\Lambda_{2}, H_{\omega}^{\alpha}\left(\Lambda_{1}\right)\right)} \leq D_{1}+D_{2},
$$

where

$$
\begin{gathered}
D_{1}=\left\|v-I_{N} v\right\|_{H^{\gamma}\left(\Lambda_{2}, H_{\omega}^{\alpha}\left(\Lambda_{1}\right)\right)}+\left\|v-I_{M} v\right\|_{H^{\gamma}\left(\Lambda_{2}, H_{\omega}^{\alpha}\left(\Lambda_{1}\right)\right)}, \\
D_{2}=\left\|\left(\mathcal{I}-I_{N}\right)\left(\mathcal{I}-I_{M}\right) v\right\|_{H^{\gamma}\left(\Lambda_{2}, H_{\omega}^{\alpha}\left(\Lambda_{1}\right)\right)} .
\end{gathered}
$$

By Theorem 2.1 and Lemma 2.9,

$$
\begin{aligned}
& D_{1} \leq c N^{\alpha+1-\frac{r}{2}}\|v\|_{H^{\gamma}\left(\Lambda_{2}, H_{\omega q}^{r}\left(\Lambda_{1}\right)\right)}+c M^{\gamma-s}\|v\|_{H^{s}\left(\Lambda_{2}, H_{\omega q}^{\alpha}\left(\Lambda_{1}\right)\right)}, \\
& D_{2} \leq c\left\|\left(\mathcal{I}-I_{M}\right) v\right\|_{H^{\gamma}\left(\Lambda_{2}, H_{\omega}^{\alpha}\left(\Lambda_{1}\right)\right)} \leq c M^{\gamma-s}\|v\|_{H^{s}\left(\Lambda_{2}, H_{\omega}^{\alpha}\left(\Lambda_{1}\right)\right)} .
\end{aligned}
$$

This completes the proof.

In the numerical analysis of the mixed Laguerre-Legendre pseudospectral method, we need the following result.

Lemma 2.11. For any $v \in C^{1}(\bar{\Omega})$ and $\phi \in V_{N, M}^{2,0}$,

$$
\left|(v, \phi)_{\omega}-(v, \phi)_{\omega, N, M}\right| \leq c\left(\left\|v-I_{N-1, M-3} v\right\|_{\omega}+\left\|v-I_{N, M} v\right\|_{\omega}\right)\|\phi\|_{\omega} .
$$

Proof. Since $I_{N-1, M-3} v \in V_{N-1, M-3}$, we have from (2.9), (2.26) and (2.32) that

$$
(v, \phi)_{\omega}-(v, \phi)_{\omega, N, M}=\left(v-I_{N-1, M-3} v, \phi\right)_{\omega}+\left(I_{N-1, M-3} v-I_{N, M} v, \phi\right)_{\omega, N, M} .
$$

Furthermore,

$$
\left|\left(v-I_{N-1, M-3} v, \phi\right)_{\omega}\right| \leq\left\|v-I_{N-1, M-3} v\right\|_{\omega}\|\phi\|_{\omega} .
$$

On the other hand, $I_{N-1, M-3} v-I_{N, M} v \in \mathcal{P}_{N}^{2,0}\left(\Lambda_{1}\right) \times P_{M}^{2,0}\left(\Lambda_{2}\right)$. Finally, using (2.30) yields that

$$
\left|\left(I_{N-1, M-3} v-I_{N, M} v, \phi\right)_{\omega, N, M}\right| \leq c\left\|\mid I_{N-1, M-3} v-I_{N, M} v\right\|_{\omega}\|\phi\|_{\omega} .
$$

This completes the proof. 


\section{The MiXed LAgUERRE-LEGENDRE PSEUdosPECTRAL SCHEME}

In this section, we construct the mixed Laguerre-Legendre pseudospectral scheme for problem (1.1), (1.2). As we know, the solution of (1.1), (1.2) possesses some conservations which come essentially from the skew-symmetricity of the nonlinear term $G(u, v)$, namely, for any $u, v \in H_{0}^{2}(\Omega)$,

$$
\iint_{\Omega} G(u, v) u d x d y=0
$$

Since the domain $\Omega$ is an infinite strip, we shall approximation problem (1.1), (1.2) by the Laguerre interpolation in the $x$-direction. It means that we multiply (1.1) by the weight $\omega(x)$ additionally. However,

$$
\iint_{\Omega} G(u, v) u \omega d x d y \neq 0 .
$$

This fact destroys the conservations. To remedy this deficiency, let $W=e^{-\frac{x}{2}} U, W_{0}$ $=e^{-\frac{x}{2}} U_{0}$ and $F=-e^{-\frac{x}{2}} f$. Then problem (1.1), (1.2) becomes

$$
\begin{cases}\partial_{t} \triangle\left(e^{-\frac{x}{2}} U\right)+G\left(e^{-\frac{x}{2}} U, e^{-\frac{x}{2}} U\right)-\mu \triangle^{2}\left(e^{-\frac{x}{2}} U\right)=-e^{-\frac{x}{2}} f, & \text { in } \Omega \times(0, T], \\ U=\frac{\partial U}{\partial n_{x}}=0, & \text { on } \partial \Omega \times[0, T], \\ \lim _{x \rightarrow \infty} e^{-\frac{x}{2}} U=\lim _{x \rightarrow \infty} e^{-\frac{x}{2}} \partial_{x} U=0, & y \in[-1,1], \\ U(x, y, 0)=e^{\frac{x}{2}} W_{0}(x, y)=U_{0}(x, y) . & t \in[0, T],\end{cases}
$$

To derive a reasonable weak formulation of $(3.2)$, let

$$
J(u, v, w)=\left(\Delta v, \partial_{y} u \partial_{x} w-\partial_{x} u \partial_{y} w\right), \quad B(u, v, w)=J\left(e^{-\frac{x}{2}} u, e^{-\frac{x}{2}} v, e^{-\frac{x}{2}} w\right) .
$$

Clearly for any $u, v, w \in H_{0}^{2}(\Omega)$,

$$
B(u, v, w)=-\left(G\left(e^{-\frac{x}{2}} u, e^{-\frac{x}{2}} v\right), e^{-\frac{x}{2}} w\right), \quad B(u, v, u)=0 .
$$

A weak formulation of $(3.2)$ is to find $U \in L^{2}\left(0, T ; H_{0, \omega}^{2}(\Omega)\right) \cap L^{\infty}\left(0, T ; H_{\omega}^{1}(\Omega)\right)$ such that

$(3.4)$

$$
\left\{\begin{array}{cc}
\left(\partial_{t} \nabla\left(e^{-\frac{x}{2}} U\right), \nabla\left(e^{-\frac{x}{2}} v\right)\right)+B(U, U, v)+\mu\left(\Delta\left(e^{-\frac{x}{2}} U\right), \Delta\left(e^{-\frac{x}{2}} v\right)\right)=(f, v)_{\omega}, \\
& \forall v \in H_{0, \omega}^{2}(\Omega), t \in(0, T], \\
U(0)=U_{0}, & \text { in } \Omega .
\end{array}\right.
$$

Due to (3.3), the solution of (3.4) keeps the related conservations.

We now construct the mixed Laguerre-Legendre pseudospectral scheme. To do this, let

$$
\begin{gathered}
a_{N, M}(u, v)=-\left(\Delta\left(e^{-\frac{x}{2}} u\right), e^{\frac{x}{2}} v\right)_{\omega, N, M}, \\
b_{N, M}(u, v)=\left(\Delta^{2}\left(e^{-\frac{x}{2}} u\right), e^{\frac{x}{2}} v\right)_{\omega, N, M}, \\
J_{N, M}(u, v, w)=\left(e^{x} \Delta v, \partial_{y} u \partial_{x} w-\partial_{x} u \partial_{y} w\right)_{\omega, N, M}, \\
B_{N, M}(u, v, w)=J_{N, M}\left(e^{-\frac{x}{2}} u, e^{-\frac{x}{2}} v, e^{-\frac{x}{2}} w\right) .
\end{gathered}
$$

It is clear that for any $u, v, w \in H_{\omega}^{2}(\Omega)$,

$$
B_{N, M}(u, v, w)+B_{N, M}(w, v, u)=0 .
$$

In particular,

$$
B_{N, M}(u, v, u)=0
$$


Obviously (3.6) simulates (3.3) properly. Thus we approximate the nonlinear term $B(u, v, w)$ by $B_{N, M}(u, v, w)$. The pseudospectral scheme for (3.3) is to find $u_{N, M}(t) \in V_{N, M}^{2,0}(\Omega)$ for all $0 \leq t \leq T$, such that

$$
\left\{\begin{array}{c}
a_{N, M}\left(\partial_{t} u_{N, M}(t), \phi\right)+B_{N, M}\left(u_{N, M}(t), u_{N, M}(t), \phi\right)+\mu b_{N, M}\left(u_{N, M}(t), \phi\right) \\
=(f(t), \phi)_{\omega, N, M}, \quad \forall \phi \in V_{N, M}^{2,0}, t \in(0, T] \\
u_{N, M}(0)=u_{0, N, M}=I_{N, M} U_{0}, \quad \text { in } \Omega_{N, M}
\end{array}\right.
$$

We now consider the stability of scheme (3.7). Assume that $u_{0, N, M}$ and $f$ have the error $\tilde{u}_{0, N, M}$ and $\tilde{f}$, respectively. They cause the error of $u_{N, M}$, denoted by $\tilde{u}_{N, M}$. In order to describe the numerical errors, we introduce the following inner products,

$$
\begin{aligned}
\tilde{a}_{N, M}(u, v)= & \int_{\Lambda_{1}} e^{-x}\left(\partial_{x} u, \partial_{x} v\right)_{M, \Lambda_{2}} d x+\int_{\Lambda_{2}}\left(\partial_{y} u, \partial_{y} v\right)_{\omega, N, \Lambda_{1}} d y \\
\tilde{b}_{N, M}(u, v)= & \int_{\Lambda_{1}} e^{-x}\left(\partial_{x}^{2} u, \partial_{x}^{2} v\right)_{M, \Lambda_{2}} d x+2 \int_{\Lambda} e^{-x} \partial_{x} \partial_{y} u \partial_{x} \partial_{y} v d x d y \\
& +\int_{\Lambda_{2}}\left(\partial_{y}^{2} u, \partial_{y}^{2} v\right)_{\omega, N, \Lambda_{1}} d y
\end{aligned}
$$

Accordingly

$$
|v|_{1, \omega, N, M, \sim}=\tilde{a}_{N, M}^{\frac{1}{2}}(v, v), \quad|v|_{2, \omega, N, M, \sim}=\tilde{b}_{N, M}^{\frac{1}{2}}(u, v) .
$$

Also let

$$
c^{*}=c^{*}\left(u_{N, M}\right)=\sup _{0 \leq t \leq T}\left|u_{N, M}\right|_{1, \omega, N, M, \sim}^{2}\left|u_{N, M}\right|_{2, \omega, N, M, \sim}^{6}+1
$$

and

$$
\rho\left(\tilde{u}_{0, N, M}, \tilde{f}, t\right)=a_{N, M}\left(\tilde{u}_{0, N, M}, \tilde{u}_{0, N, M}\right)+\int_{0}^{T}\|\tilde{f}(\eta)\|_{\omega, N, M}^{2} d \eta .
$$

We have the following result.

Theorem 3.1. For any $0 \leq t \leq T$,

$$
\left|\tilde{u}_{N, M}(t)\right|_{1, \omega, N, M, \sim}^{2}+\mu \int_{0}^{t}\left|\tilde{u}_{N, M}(\eta)\right|_{2, \omega, N, M, \sim}^{2} d \eta \leq c e^{\frac{c c^{*}}{\mu} t} \rho\left(\tilde{u}_{0, N, M}, \tilde{f}, t\right) .
$$

We next deal with the convergence of scheme (3.7). For simplicity, let

$$
\begin{aligned}
\tilde{M}_{q}^{r, s}(\Omega)= & L^{2}\left(\Lambda_{2}, H_{\omega^{q}}^{r}\left(\Lambda_{1}\right)\right) \cap H^{s}\left(\Lambda_{2}, L_{\omega^{q}}^{2}\left(\Lambda_{1}\right)\right) \\
& \cap H^{1}\left(\Lambda_{2}, H_{\omega^{q}}^{r-1}\left(\Lambda_{1}\right)\right) \cap H^{s-1}\left(\Lambda_{2}, H_{\omega^{q}}^{1}\left(\Lambda_{1}\right)\right) \\
& \cap H^{2}\left(\Lambda_{2}, H_{\omega^{q}}^{r-2}\left(\Lambda_{1}\right)\right) \cap H^{s-2}\left(\Lambda_{2}, H_{\omega^{q}}^{2}\left(\Lambda_{1}\right)\right), \quad q>0, r, s \geq 2 .
\end{aligned}
$$

Its norm $\|v\|_{\tilde{M}_{q}^{r, s}}$ is defined in the same way as for $\|v\|_{M_{q}^{r, s}}$ in Section 2. Clearly for any $0<q_{1}<q$,

$$
\|v\|_{M_{q}^{r, s}} \leq c\|v\|_{\tilde{M}_{1}^{r, s}} .
$$

Also for $q>0, r, s \geq 0$ and $\delta>0$,

$$
\begin{aligned}
Y_{q, \delta}^{r, s}(\Omega)= & H_{\omega}^{\left[\frac{r+1}{2}\right]+2+\delta}(\Omega) \cap H_{\omega}^{\left[\frac{s+1}{2}\right]+2+\delta}(\Omega) \cap L^{2}\left(\Lambda_{2}, H_{\omega^{q}}^{r+2}\left(\Lambda_{1}\right)\right) \\
& \cap H^{s+2}\left(\Lambda_{2}, L_{\omega^{q}}^{2}\left(\Lambda_{1}\right)\right) \cap H^{2}\left(\Lambda_{2}, H_{\omega^{q}}^{r}\left(\Lambda_{1}\right)\right) \cap H^{s}\left(\Lambda_{2}, H_{\omega^{q}}^{2}\left(\Lambda_{1}\right)\right)
\end{aligned}
$$

with the norm $\|v\|_{Y_{q, \delta}^{r, s}}$. 
Theorem 3.2. Assume that for $0<q<1, r \geq 4, s \geq 2, \tilde{r}, \tilde{s} \geq 0, r_{1}, s_{1} \geq$ $2, r_{0}, s_{0} \geq 2, \lambda, \gamma \geq 0$ and $\delta>0$

$$
\begin{gathered}
U \in L^{2}\left(0, T ; \tilde{M}_{q}^{r, s}(\Omega)\right) \cap L^{4}\left(0, T ; Y_{q, \delta}^{\tilde{r}, \tilde{s}}(\Omega)\right) \\
\cap L^{\infty}\left(0, T ; M_{q}^{2,2}(\Omega) \cap H^{3+\delta}(\Omega)\right) \cap H^{1}\left(0, T ; M_{q}^{r_{1}, s_{1}}(\Omega)\right), \\
\quad U_{0} \in L^{2}\left(0, T ; \tilde{M}_{q}^{r_{0}, s_{0}}(\Omega)\right), \\
\quad f \in L^{2}\left(0, T ; H^{\gamma}\left(\Lambda_{2}, L_{\omega^{q}}^{2}\left(\Lambda_{1}\right)\right) \cap L^{2}\left(\Lambda_{2}, H_{\omega^{q}}^{\lambda}\left(\Lambda_{1}\right)\right)\right) .
\end{gathered}
$$

Then for all $0 \leq t \leq T$,

$$
\begin{aligned}
\mid U(t) & -\left.u_{N, M}(t)\right|_{1, \omega, N, M, \sim} ^{2}+\mu \int_{0}^{t}\left|U(\eta)-u_{N, M}(\eta)\right|_{2, \omega, N, M, \sim}^{2} d \eta \\
& \leq c^{*}\left(\left(N^{1-\frac{r}{2}}+M^{2-s}\right)^{2} \max (N, M)\right. \\
& +\left(N^{2-\frac{r}{2}}+M^{2-s}\right)^{2}+\left(N^{-\frac{\tilde{r}}{2}}+M^{-\tilde{s}}\right)^{2}+\left(N^{1-\frac{r_{1}}{2}}+M^{2-s_{1}}\right)^{2}\left(N^{-1}+M^{-2}\right) \\
& \left.+\left(N^{2-\frac{r_{0}}{2}}+M^{1-s_{0}}\right)^{2}+\left(N^{1-\frac{r_{0}}{2}}+M^{2-s_{0}}\right)^{2}\left(N^{-1}+M^{-2}\right)+\left(N^{-\frac{\lambda}{2}}+M^{-\gamma}\right)^{2}\right),
\end{aligned}
$$

with $c^{*}$ being a positive constant depending on the norms of the spaces mentioned of $U, U_{0}$ and $f$.

Remark 3.1. If $N=O\left(M^{2}\right), \tilde{r}, \lambda \geq r-4, \tilde{s}, \gamma \geq s-3, r_{0} \geq r, r_{1} \geq r-3$ and $s_{0}, s_{1} \geq s-2$, then

$\left|U(t)-u_{N, M}(t)\right|_{1, \omega, N, M, \sim}^{2}+\mu \int_{0}^{t}\left|U(\eta)-u_{N, M}(\eta)\right|_{2, \omega, N, M, \sim}^{2} d \eta \leq c N^{\max \left(4-r, \frac{3}{2}-\frac{s}{2}\right)}$.

If $N=O(M), \tilde{r}, \lambda \geq r-4, \tilde{s}, \gamma \geq s-2, r_{0} \geq r, r_{1} \geq r-3, s_{0} \geq s-1$ and $s_{1} \geq s-\frac{1}{2}$, then the power of $N$ in the above estimate becomes $\max \left(4-r, 5-2 s, 4-\frac{r}{2}-s\right)$. Clearly we get a better convergence rate when $N=O\left(M^{2}\right)$. This feature comes from the fact that the convergence rate of the Laguerre approximation is only half of that for the Legendre approximation.

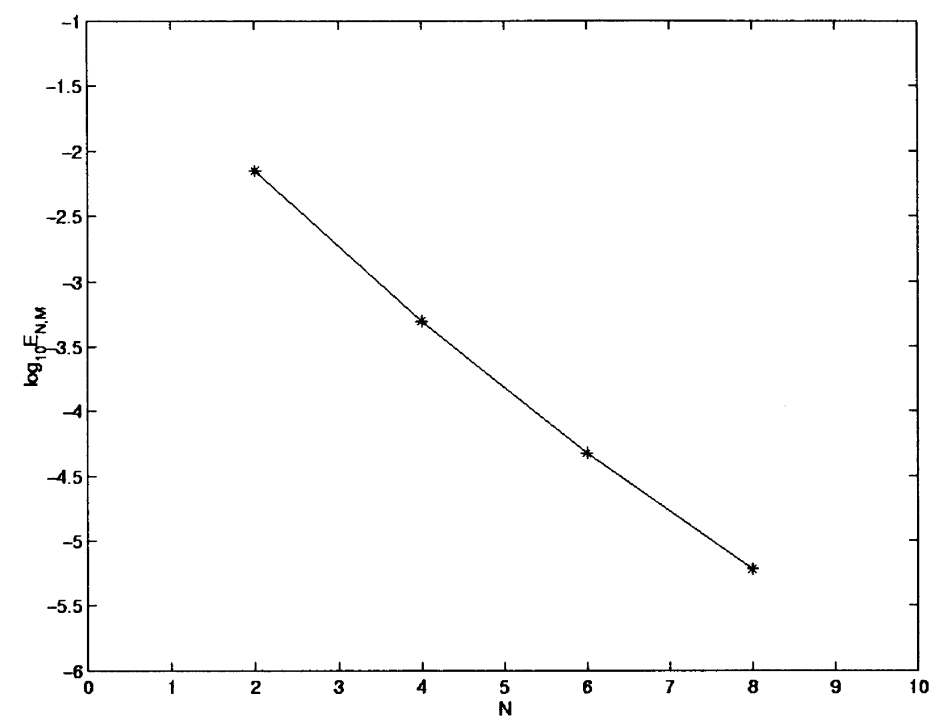

Figure 1. Convergence rate for $N=M^{2}$ 


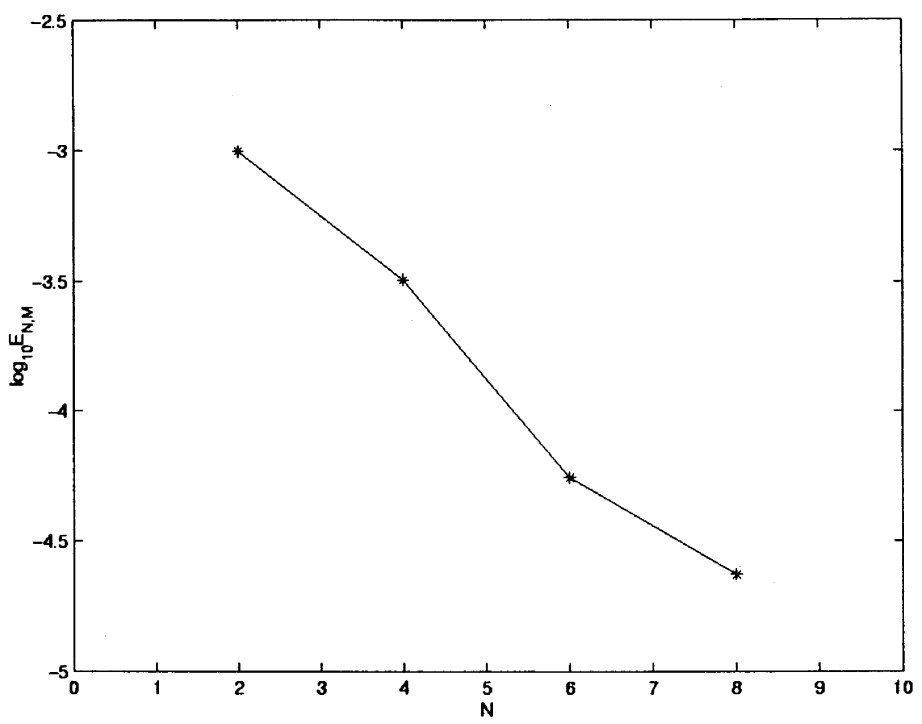

Figure 2. Convergence rate for $N=M$

We shall prove Theorems 3.1 and 3.2 in the next section.

In the end of this section, we give some numerical results. We take the test function

$$
U(x, y, t)=\frac{x^{2}\left(1-y^{2}\right)^{2} \sin (k x t+k y t)}{(2+x+y)^{h}}
$$

with $k=0.2, h=5.0$. In actual computation, we use the standard fourth-order Runge-Kutta method in time $t$ with the step $\tau$. Let

$$
E_{N, M}(t)=\left\|U(t)-u_{N, M}(t)\right\|_{\omega, N, M}
$$

and

$$
\tilde{E}_{N, M}(t)=\frac{\left\|U(t)-u_{N, M}(t)\right\|_{\omega, N, M}}{\|U(t)\|_{\omega, N, M}}
$$

The errors $E_{N, M}(t)$ and $\tilde{E}_{N, M}(t)$ at $t=1$ are listed in Tables 1 and 2, which indicate the convergence of scheme (3.7) as $N, M$ increase and $\tau$ decreases. The errors $E_{N, M}(t)$ and $\tilde{E}_{N, M}(t)$ with $M=16, N=64$ and $\tau=0.001$ listed in Table 3 show the stability of calculation. For checking the spectral accuracy in the space, we list the errors $E_{N, M}(1)$ with $N=M^{2}$ and $\tau=0.001$ in Figure 1, while we list errors $E_{N, M}(1)$ with $N=M$ and $\tau=0.001$ in Figure 22 Both show the spectral accuracy. But the the errors with $N=M^{2}$ are smaller than those with $N=M$. This coincides with the theoretical analysis. 
TABLE 1. The errors $E_{N, M}(1)$.

\begin{tabular}{|c|c|c|c|c|}
\hline$\tau$ & $N=4, M=2$ & $N=16, M=4$ & $N=36, M=6$ & $N=64, M=8$ \\
\hline 0.1 & $4.921 \mathrm{E}-02$ & $3.255 \mathrm{E}-03$ & $2.836 \mathrm{E}-03$ & $1.785 \mathrm{E}-04$ \\
\hline 0.01 & $2.219 \mathrm{E}-02$ & $2.785 \mathrm{E}-03$ & $2.644 \mathrm{E}-04$ & $1.859 \mathrm{E}-05$ \\
\hline 0.001 & $7.005 \mathrm{E}-03$ & $4.953 \mathrm{E}-04$ & $4.741 \mathrm{E}-05$ & $6.023 \mathrm{E}-06$ \\
\hline
\end{tabular}

TABLE 2. The errors $\tilde{E}_{N, M}(1)$.

\begin{tabular}{|c|c|c|c|c|}
\hline$\tau$ & $N=4, M=2$ & $N=16, M=4$ & $N=36, M=6$ & $N=64, M=8$ \\
\hline 0.1 & $6.195 \mathrm{E}-01$ & $1.014 \mathrm{E}-01$ & $8.298 \mathrm{E}-02$ & $5.604 \mathrm{E}-02$ \\
\hline 0.01 & $2.729 \mathrm{E}-01$ & $8.681 \mathrm{E}-02$ & $8.318 \mathrm{E}-03$ & $5.835 \mathrm{E}-03$ \\
\hline 0.001 & $2.466 \mathrm{E}-01$ & $1.686 \mathrm{E}-02$ & $1.629 \mathrm{E}-03$ & $2.036 \mathrm{E}-04$ \\
\hline
\end{tabular}

TABLE 3 . The errors $E_{N, M}(t)$ and $\tilde{E}_{N, M}(t)$.

\begin{tabular}{|c|c|c|}
\hline$t$ & $E_{N, M}(t)$ & $\tilde{E}_{N, M}(t)$ \\
\hline 1.0 & $6.023 \mathrm{E}-06$ & $2.036 \mathrm{E}-04$ \\
\hline 2.0 & $6.128 \mathrm{E}-06$ & $2.614 \mathrm{E}-04$ \\
\hline 3.0 & $9.839 \mathrm{E}-06$ & $6.968 \mathrm{E}-04$ \\
\hline 4.0 & $1.060 \mathrm{E}-05$ & $1.047 \mathrm{E}-03$ \\
\hline 5.0 & $1.459 \mathrm{E}-05$ & $1.370 \mathrm{E}-03$ \\
\hline
\end{tabular}

\section{The PRoof of ERRor ESTIMATES}

We need some preparations stated in the following six lemmas.

Lemma 4.1. Let $\phi \in V_{N, M}^{2,0}$. We have that

(i) $c_{1} \min \left(\frac{1}{N}, \frac{1}{M}\right)|\phi|_{1, \omega}^{2} \leq c_{2}|\phi|_{1, \omega, N, M, \sim}^{2} \leq a_{N, M}(\phi, \phi) \leq c_{3}|\phi|_{1, \omega, N, M, \sim}^{2} \leq c_{4}|\phi|_{1, \omega}^{2}$,

(ii) $c_{1} \min \left(\frac{1}{N}, \frac{1}{M}\right)|\phi|_{2, \omega}^{2} \leq|\phi|_{2, \omega, N, M, \sim}^{2} \leq c_{2}|\phi|_{2, \omega}^{2}$,

(iii) $c\|\phi\|_{\omega}^{2} \leq \frac{1}{3}|\phi|_{1, \omega}^{2} \leq|\phi|_{2, \omega, N, M, \sim}^{2}$.

Proof. By (2.9), Lemma 2.8 and integration by parts, we deduce that

$$
\begin{aligned}
a_{N, M}(\phi, \phi) & =-\left(\partial_{x}^{2} \phi+\partial_{y}^{2} \phi-\partial_{x} \phi+\frac{1}{4} \phi, \phi\right)_{\omega, N, M} \\
& \geq \int_{\Lambda_{1}} e^{-x}\left\|\partial_{x} \phi\right\|_{M, \Lambda_{2}}^{2} d x+\int_{\Lambda_{2}}\left\|\partial_{y} \phi\right\|_{\omega, N, \Lambda_{1}}^{2} d y-\frac{1}{4}\|\phi\|_{\omega, N, M}^{2} .
\end{aligned}
$$

By the Poincaré inequality, for any $v \in H_{0}^{1}\left(\Lambda_{2}\right)$,

$$
\|v\|_{\Lambda_{2}}^{2} \leq 2\left\|\partial_{y} v\right\|_{\Lambda_{2}}^{2}
$$

So by Lemma 2.7,

$$
\int_{\Lambda_{2}}\left\|\partial_{y} \phi\right\|_{\omega, N, \Lambda_{1}}^{2} d y \geq \frac{1}{2 c}\|\phi\|_{\omega, N, M}^{2}
$$


By $\mathrm{Xu}$ and Guo [22], for any $v \in H_{0, \omega}^{1}\left(\Lambda_{1}\right)$,

$$
\|v\|_{\omega, \Lambda_{1}}^{2} \leq 4\left\|\partial_{x} v\right\|_{\omega, \Lambda_{1}}^{2} .
$$

So by Lemma 2.4,

$$
\int_{\Lambda_{1}} e^{-x}\left\|\partial_{x} \phi\right\|_{M, \Lambda_{2}}^{2} d x \geq \frac{1}{4}\|\phi\|_{\omega, N, M}^{2}
$$

Take $c_{2}=\frac{2}{2+c}$. Then the previous statements lead to

$$
\left(1-c_{2}\right)\left(\int_{\Lambda_{1}} e^{-x}\left\|\partial_{x} \phi\right\|_{M, \Lambda_{2}}^{2} d x+\int_{\Lambda_{2}}\left\|\partial_{y} \phi\right\|_{\omega, N, \Lambda_{1}}^{2} d y\right) \geq \frac{1}{4}\|\phi\|_{\omega, N, M}^{2} .
$$

Thus (4.1) reads

$$
a_{N, M}(\phi, \phi) \geq c_{2} \int_{\Lambda_{1}} e^{-x}\left\|\partial_{x} \phi\right\|_{M, \Lambda_{2}}^{2} d x+c_{2} \int_{\Lambda_{2}}\left\|\partial_{y} \phi\right\|_{\omega, N, \Lambda_{1}}^{2} d y=c_{2}|\phi|_{1, \omega, N, M, \sim}^{2} .
$$

Next, by virtue of Lemmas 2.4 and 2.7,

$$
c_{2}|\phi|_{1, \omega, N, M, \sim}^{2} \geq c_{1} \min \left(\frac{1}{N}, \frac{1}{M}\right)|\phi|_{1, \omega}^{2} .
$$

Furthermore, we obtain from (4.1) and Lemmas 2.4, 2.7 and 2.8 that

$$
\begin{aligned}
a_{N, M}(\phi, \phi) & \leq \int_{\Lambda_{1}} e^{-x}\left\|\partial_{x} \phi\right\|_{M, \Lambda_{2}}^{2} d x+c \int_{\Lambda_{2}}\left\|\partial_{y} \phi\right\|_{\omega, N, \Lambda_{1}}^{2} d y \\
& \leq c_{3}|\phi|_{1, \omega, N, M, \sim}^{2} \leq c_{4}|\phi|_{1, \omega}^{2} .
\end{aligned}
$$

The previous statements imply result (i). Result (ii) comes from Lemmas 2.4 and 2.7. Finally, result (iii) comes from (4.2), (4.3) and the definition of $|\phi|_{2, \omega, N, M, \sim \text {. }}$

Lemma 4.2. For any $\phi \in C^{1}\left(0, T ; V_{N, M}^{2,0}\right)$,

$$
a_{N, M}\left(\partial_{t} \phi, \phi\right)=\frac{1}{2} \frac{d}{d t} a_{N, M}(\phi, \phi) .
$$

Proof. A direct calculation gives that

$$
a_{N, M}\left(\partial_{t} \phi, \phi\right)=A_{1}+A_{2}
$$

with

$$
\begin{gathered}
A_{1}=-\left(\partial_{x}^{2} \partial_{t} \phi, \phi\right)_{\omega, N, M}+\left(\partial_{x} \partial_{t} \phi, \phi\right)_{\omega, N, M}-\frac{1}{4}\left(\partial_{t} \phi, \phi\right)_{\omega, N, M}, \\
A_{2}=-\left(\partial_{y}^{2} \partial_{t} \phi, \phi\right)_{\omega, N, M} .
\end{gathered}
$$

By (2.9) and integration by parts,

$$
A_{1}=\frac{1}{2} \frac{d}{d t}\left(\int_{\Lambda_{1}} e^{-x}\left\|\partial_{x} \phi\right\|_{M, \Lambda_{2}}^{2} d x-\frac{1}{4}\|\phi\|_{\omega, N, M}^{2}\right) .
$$

On the other hand, as pointed out on page 92 of Bernardi and Maday [3], for any $\psi_{1}, \psi_{2} \in \mathcal{P}_{M}^{2,0}\left(\Lambda_{2}\right)$,

Thus

$$
\left(\partial_{y}^{2} \psi_{1}, \psi_{2}\right)_{M, \Lambda_{2}}=\left(\psi_{1}, \partial_{y}^{2} \psi_{2}\right)_{M, \Lambda_{2}} .
$$

The combination of (4.1) and (4.4)-(4.6) implies the desired result. 
Lemma 4.3. For any $\phi \in V_{N, M}^{2,0}$,

$$
b_{N, M}(\phi, \phi) \geq|\phi|_{2, \omega, N, M, \sim}^{2}-c|\phi|_{1, \omega, N, M, \sim}^{2} .
$$

Proof. We have

$$
\begin{aligned}
b_{N, M}(\phi, \phi)= & \left(\partial_{x}^{4}\left(e^{-\frac{x}{2}} \phi\right), e^{\frac{x}{2}} \phi\right)_{\omega, N, M}+2\left(\partial_{x}^{2} \partial_{y}^{2}\left(e^{-\frac{x}{2}} \phi\right), e^{\frac{x}{2}} \phi\right)_{\omega, N, M} \\
& +\left(\partial_{y}^{4}\left(e^{-\frac{x}{2}} \phi\right), e^{\frac{x}{2}} \phi\right)_{\omega, N, M} .
\end{aligned}
$$

So it remains to estimate the terms at the right side of (4.7). First, integration by parts gives that for any $v \in H_{0, \omega}^{1}\left(\Lambda_{1}\right)$,

$$
\int_{\Lambda_{1}}\left(\partial_{x}\left(e^{-\frac{x}{2}} v\right)\right)^{2} d x=\int_{\Lambda_{1}} e^{-x}\left(\left(\partial_{x} v\right)^{2}-\frac{1}{4} v^{2}\right) d x
$$

and for any $v \in H_{0, \omega}^{2}\left(\Lambda_{1}\right)$,

$$
\int_{\Lambda_{1}}\left(\partial_{x}^{2}\left(e^{-\frac{x}{2}} v\right)\right)^{2} d x=\int_{\Lambda_{1}} e^{-x}\left(\left(\partial_{x}^{2} v\right)^{2}-\frac{1}{2}\left(\partial_{x} v\right)^{2}+\frac{1}{16} v^{2}\right) d x
$$

Now let

$$
\psi(x, y)=\partial_{x}^{4}\left(e^{-\frac{x}{2}} \phi(x, y)\right)-\frac{1}{16} e^{-\frac{x}{2}} \phi(x, y) .
$$

Since $e^{\frac{x}{2}} \phi \psi \in \mathcal{P}_{2 N-1}^{2,0} \times \mathcal{P}_{2 M}^{2,0}\left(\Lambda_{2}\right),(2.9)$ and integration by parts lead to

$$
\left(\psi, e^{\frac{x}{2}} \phi\right)_{N, \Lambda_{1}}=\int_{\Lambda_{1}}\left(\partial_{x}^{2}\left(e^{-\frac{x}{2}} \phi\right)\right)^{2} d x-\frac{1}{16} \int_{\Lambda_{1}} \phi^{2} e^{-x} d x
$$

Therefore by (4.9),

$$
\begin{aligned}
& \left(\partial_{x}^{4}\left(e^{-\frac{x}{2}} \phi\right), e^{\frac{x}{2}} \phi\right)_{N, M} \\
& \quad=\left(\psi, e^{\frac{x}{2}} \phi\right)_{\omega, N, M}+\frac{1}{16}\|\phi\|_{\omega, N, M}^{2} \\
& \quad=\int_{\Lambda_{1}} e^{-x}\left\|\partial_{x}^{2} \phi\right\|_{M, \Lambda_{2}}^{2} d x-\frac{1}{2} \int_{\Lambda_{1}} e^{-x}\left\|\partial_{x} \phi\right\|_{M, \Lambda_{2}}^{2} d x+\frac{1}{16}\|\phi\|_{\omega, N, M}^{2} .
\end{aligned}
$$

Next, by (2.9), Lemma 2.8 and integration by parts,

$$
\begin{aligned}
\left(\partial_{x}^{2} \partial_{y}^{2}\left(e^{-\frac{x}{2}} \phi\right), e^{\frac{x}{2}} \phi\right)_{\omega, N, M} & \\
= & \int_{\Lambda_{1}} e^{-x}\left(\partial_{x}^{2} \partial_{y}^{2}\left(e^{-\frac{x}{2}} \phi\right)-\frac{1}{4} e^{-\frac{x}{2}} \partial_{y}^{2} \phi, e^{\frac{x}{2}} \phi\right)_{M, \Lambda_{2}} d x+\frac{1}{4}\left(\partial_{y}^{2} \phi, \phi\right)_{\omega, N, M} \\
(4.11)= & -\int_{\Lambda_{1}}\left(\partial_{y}^{2} \partial_{x}\left(e^{-\frac{x}{2}} \phi\right), \partial_{x}\left(e^{-\frac{x}{2}} \phi\right)\right)_{M, \Lambda_{2}} d x-\frac{1}{4} \int_{\Lambda_{1}} e^{-x}\left(\partial_{y}^{2} \phi, \phi\right)_{M, \Lambda_{2}} d x \\
& +\frac{1}{4}\left(\partial_{y}^{2} \phi, \phi\right)_{\omega, N, M} \\
\geq & \int_{\Omega} e^{-x}\left(\partial_{x} \partial_{y} \phi\right)^{2} d x d y-\frac{c}{4} \int_{\Lambda_{2}}\left\|\partial_{y} \phi\right\|_{\omega, N, \Lambda_{1}}^{2} d y
\end{aligned}
$$

By (2.26) and integration by parts, we have

$$
\left(\partial_{y}^{4}\left(e^{-\frac{x}{2}} \phi\right), e^{\frac{x}{2}} \phi\right)_{\omega, N, M}=\int_{\Lambda_{2}}\left(\partial_{y}^{4} \phi, \phi\right)_{\omega, N, \Lambda_{1}} d y=\int_{\Lambda_{2}}\left\|\partial_{y}^{2} \phi\right\|_{\omega, N, \Lambda_{1}}^{2} d y
$$

By substituting (4.10)-(4.12) into (4.7), we reach the desired result. 
Lemma 4.4. For any $\phi \in V_{N, M}^{2,0}$,

$$
\begin{aligned}
& \sum_{i=1}^{N-1} \sum_{j=1}^{M-3} \omega_{i j}^{N, M} e^{\sigma_{i}^{N}}\left(\left(\partial_{x}\left(e^{-\frac{x}{2}} \phi\right)\left(\sigma_{i}^{N}, \tau_{j}^{M}\right)\right)^{4}+\left(\partial_{y}\left(e^{-\frac{x}{2}} \phi\right)\left(\sigma_{i}^{N}, \tau_{j}^{M}\right)\right)^{4}\right) \\
& \quad \leq c|\phi|_{1, \omega, N, M, \sim}|\phi|_{2, \omega, N, M, \sim}^{3} .
\end{aligned}
$$

Proof. We first derive some inequalities. By (4.8), (4.9) and integration by parts, we have that for any $v \in H_{0, \omega}^{1}\left(\Lambda_{1}\right)$,

$$
\left\|\partial_{x}\left(e^{-\frac{x}{2}} v\right)\right\|_{\Lambda_{1}}^{2} \leq\left\|\partial_{x} v\right\|_{\omega, \Lambda_{1}}^{2}
$$

and for any $v \in H_{0, \omega}^{2}\left(\Lambda_{1}\right)$,

$$
\left\|\partial_{x}^{2}\left(e^{-\frac{x}{2}} v\right)\right\|_{\Lambda_{1}}^{2}=\left\|\partial_{x}^{2} v\right\|_{\omega, \Lambda_{1}}^{2}-\frac{1}{4}\left\|\partial_{x}\left(e^{-\frac{x}{2}} v\right)\right\|_{\Lambda_{1}}^{2}-\frac{1}{4}\left\|\partial_{x} v\right\|_{\omega, \Lambda_{1}}^{2} \leq\left\|\partial_{x}^{2} v\right\|_{\omega, \Lambda_{1}}^{2}
$$

Next, if $\partial_{x} v \in H_{0}^{1}\left(\Lambda_{2}\right)$ for all $x \in \Lambda_{1}$, then by (4.2),

$$
\left\|\partial_{x} v\right\|_{\Lambda_{2}}^{2} \leq 2\left\|\partial_{x} \partial_{y} v\right\|_{\Lambda_{2}}^{2} .
$$

Furthermore, we have from (2.9), Lemma 2.4 and integration by parts that for any $\psi \in \mathcal{P}_{N}^{2,0}\left(\Lambda_{1}\right)$.

$$
\begin{aligned}
& \sum_{i=1}^{N-1} \omega_{i}^{N} e^{\sigma_{i}^{N}}\left(\partial_{x}\left(e^{-\frac{x}{2}} \psi\right)\left(\sigma_{i}^{N}\right)\right)^{2} \\
& \quad=\int_{\Lambda_{1}} e^{-x}\left(\partial_{x} \psi\right)^{2} d x-\frac{1}{2} \int_{\Lambda_{1}} e^{-x} \psi^{2} d x+\frac{1}{4} \sum_{i=1}^{N-1} \omega_{i}^{N} \psi^{2}\left(\sigma_{i}^{N}\right) \\
& \quad \leq\left\|\partial_{x} \psi\right\|_{\omega, \Lambda_{1}}^{2} .
\end{aligned}
$$

Also by the Cauchy inequality, (4.3), Lemma 2.4 and Remarks 2.2 and 2.3,

$$
\begin{aligned}
& \sum_{i=1}^{N-1} \omega_{i}^{N} e^{\sigma_{i}^{N}}\left(\partial_{x}^{2}\left(e^{-\frac{x}{2}} \psi\right)\left(\sigma_{i}^{N}\right)\right)^{2} \\
& \quad \leq 3 \sum_{i=1}^{N-1} \omega_{i}^{N}\left(\left(\partial_{x}^{2} \psi\left(\sigma_{i}^{N}\right)\right)^{2}+\left(\partial_{x} \psi\left(\sigma_{i}^{N}\right)\right)^{2}+\frac{1}{16} \psi^{2}\left(\sigma_{i}^{N}\right)\right) \\
& \quad \leq c\left\|\partial_{x}^{2} \psi\right\|_{\omega, \Lambda_{1}}^{2} .
\end{aligned}
$$

Now we observe that

$$
\begin{aligned}
& \left(\partial_{x}\left(e^{-\frac{x}{2}} \phi\right)\left(\sigma_{i}^{N}, \tau_{j}^{M}\right)\right)^{2} \leq 2 \int_{\Lambda_{1}}\left|\partial_{x}\left(e^{-\frac{x}{2}} \phi\right)\left(x, \tau_{j}^{M}\right)\right|\left|\partial_{x}^{2}\left(e^{-\frac{x}{2}} \phi\right)\left(x, \tau_{j}^{M}\right)\right| d x \\
& \left(\partial_{x}\left(e^{-\frac{x}{2}} \phi\right)\left(\sigma_{i}^{N}, \tau_{j}^{M}\right)\right)^{2} \leq 2 \int_{\Lambda_{2}}\left|\partial_{x}\left(e^{-\frac{x}{2}} \phi\right)\left(\sigma_{i}^{N}, y\right)\right|\left|\partial_{x} \partial_{y}\left(e^{-\frac{x}{2}} \phi\right)\left(\sigma_{i}^{N}, y\right)\right| d y .
\end{aligned}
$$


Thus by (4.15)-(4.17) and Remark 2.4, we obtain that

$$
\begin{aligned}
\sum_{i=1}^{N-1} & \sum_{j=1}^{M-3} \omega_{i j}^{N, M} e^{\sigma_{i}^{N}}\left(\partial_{x}\left(e^{-\frac{x}{2}} \phi\right)\left(\sigma_{i}^{N}, \tau_{j}^{M}\right)\right)^{4} \\
\leq & 4 \int_{\Lambda_{1}} \sum_{j=1}^{M-3} \rho_{j}^{M}\left|\partial_{x}\left(e^{-\frac{x}{2}} \phi\right)\left(x, \tau_{j}^{M}\right)\right|\left|\partial_{x}^{2}\left(e^{-\frac{x}{2}} \phi\right)\left(x, \tau_{j}^{M}\right)\right| d x \\
& \cdot \int_{\Lambda_{2}} \sum_{i=1}^{N-1} \omega_{i}^{N} e^{\sigma_{i}^{N}}\left|\partial_{x}\left(e^{-\frac{x}{2}} \phi\right)\left(\sigma_{i}^{N}, y\right)\right|\left|\partial_{x} \partial_{y}\left(e^{-\frac{x}{2}} \phi\right)\left(\sigma_{i}^{N}, y\right)\right| d y \\
\leq & \left.4 \int_{\Lambda_{1}} \sum_{j=1}^{M-3} \rho_{j}^{M}\left(\partial_{x}\left(e^{-\frac{x}{2}} \phi\right)\left(x, \tau_{j}^{M}\right)\right)^{2} d x\right)^{\frac{1}{2}} \\
& \cdot\left(\int_{\Lambda_{1}} \sum_{j=1}^{M-3} \rho_{j}^{M}\left(\partial_{x}^{2}\left(e^{-\frac{x}{2}} \phi\right)\left(x, \tau_{j}^{M}\right)\right)^{2} d x\right)^{\frac{1}{2}} \\
& \left(\int_{\Lambda_{2}} \sum_{i=1}^{N-1} \omega_{i}^{N} e^{\sigma_{i}^{N}}\left(\partial_{x}\left(e^{-\frac{x}{2}} \phi\right)\left(\sigma_{i}^{N}, y\right)\right)^{2} d y\right)^{\frac{1}{2}} \\
& \left(\int_{\Lambda_{2}} \sum_{i=1}^{N-1} \omega_{i}^{N} e^{\sigma_{i}^{N}}\left(\partial_{x} \partial_{y}\left(e^{-\frac{x}{2}} \phi\right)\left(\sigma_{i}^{N}, y\right)\right)^{2} d y\right)^{\frac{1}{2}} \\
\leq & c|\phi|_{1, \omega, N, M, \sim}|\phi|_{2, \omega, N, M, \sim}\left\|\partial_{x} \partial_{y} \phi\right\|_{\omega}^{2} \leq c|\phi|_{1, \omega, N, M, \sim|\phi|_{2, \omega, N, M, \sim}^{3}}
\end{aligned}
$$

Next we have that

$$
\begin{gathered}
\left(\partial_{y}\left(e^{-\frac{x}{2}} \phi\right)\left(\sigma_{i}^{N}, \tau_{j}^{M}\right)\right)^{2} \leq 2 \int_{\Lambda_{1}}\left|\partial_{y}\left(e^{-\frac{x}{2}} \phi\right)\left(x, \tau_{j}^{M}\right)\right|\left|\partial_{x} \partial_{y}\left(e^{-\frac{x}{2}} \phi\right)\left(x, \tau_{j}^{M}\right)\right| d x \\
\left(\partial_{y}\left(e^{-\frac{x}{2}} \phi\right)\left(\sigma_{i}^{N}, \tau_{j}^{M}\right)\right)^{2} \leq 2 \int_{\Lambda_{2}}\left|\partial_{y}\left(e^{-\frac{x}{2}} \phi\right)\left(\sigma_{i}^{N}, y\right)\right|\left|\partial_{y}^{2}\left(e^{-\frac{x}{2}} \phi\right)\left(\sigma_{i}^{N}, y\right)\right| d y
\end{gathered}
$$

By an argument similar to that used in the derivation of (4.18), we use Remark 2.4 and (4.2) to deduce that

$$
\sum_{i=1}^{N-1} \sum_{j=1}^{M-3} \omega_{i j}^{N, M} e^{\sigma_{i}^{N}}\left(\partial_{y}\left(e^{-\frac{x}{2}} \phi\right)\left(\sigma_{i}^{N}, \tau_{j}^{M}\right)\right)^{4} \leq c|\phi|_{1, \omega, N, M, \sim}|\phi|_{2, \omega, N, M, \sim}^{3} .
$$

Finally the desired result follows from (4.18) and (4.19).

Lemma 4.5. For any $\phi \in V_{N, M}^{2,0}$,

$$
\sum_{i=1}^{N-1} \sum_{j=1}^{M-3} \omega_{i j}^{N, M} e^{\sigma_{i}^{N}}\left(\Delta\left(e^{-\frac{x}{2}} \phi\right)\left(\sigma_{i}^{N}, \tau_{j}^{M}\right)\right)^{2} \leq c|\phi|_{2, \omega, N, M, \sim}^{2}
$$


Proof. We have from (4.16) and Remark 2.5 that

$$
\begin{aligned}
& \sum_{i=1}^{N-1} \sum_{j=1}^{M-3} \omega_{i j}^{N, M} e^{\sigma_{i}^{N}}\left(\Delta\left(e^{-\frac{x}{2}} \phi\right)\left(\sigma_{i}^{N}, \tau_{j}^{M}\right)\right)^{2} \\
& \quad \leq c \sum_{j=1}^{M-3} \rho_{j}^{M} \int_{\Lambda_{1}} e^{-x}\left(\partial_{x}^{2} \phi\left(x, \tau_{j}^{M}\right)\right)^{2} d x+c \sum_{i=1}^{N-1} \omega_{i}^{N} \int_{\Lambda_{2}}\left(\partial_{y}^{2} \phi\left(\sigma_{i}^{N}, y\right)\right)^{2} d y \\
& \quad=c|\phi|_{2, \omega, N, M, \sim}^{2} .
\end{aligned}
$$

Lemma 4.6. For any $\phi, \psi \in V_{N, M}^{2,0}$,

$$
\left|B_{N, M}(\phi, \phi, \psi)\right| \leq c|\phi|_{1, \omega, N, M, \sim}^{\frac{1}{4}}|\psi|_{1, \omega, N, M, \sim}^{\frac{1}{4}}|\phi|_{2, \omega, N, M, \sim}^{\frac{7}{4}}|\psi|_{2, \omega, N, M, \sim}^{\frac{3}{4}} .
$$

Proof. The conclusion comes from the Hölder inequality and Lemmas 4.4 and 4.5.

We now prove Theorem 3.1. By (3.7), the error $\tilde{u}_{N, M}$ satisfies

$$
\left\{\begin{array}{l}
a_{N, M}\left(\partial_{t} \tilde{u}_{N, M}(t), \phi\right)+B_{N, M}\left(\tilde{u}_{N, M}(t), u_{N, M}(t)+\tilde{u}_{N, M}(t), \phi\right) \\
+B_{N, M}\left(u_{N, M}(t), \tilde{u}_{N, M}(t), \phi\right)+\mu b_{N, M}\left(\tilde{u}_{N, M}, \phi\right) \\
\quad=(\tilde{f}(t), \phi)_{\omega, N, M}, \quad \forall \phi \in V_{N, M}^{2,0}, t \in(0, T] . \\
\tilde{u}_{N, M}(0)=\tilde{u}_{0, N, M} .
\end{array}\right.
$$

Letting $\phi=2 \tilde{u}_{N, M}$ in (4.20), we get from (3.5), (3.6) and Lemma 4.2 that

$$
\begin{aligned}
& \frac{d}{d t} a_{N, M}\left(\tilde{u}_{N, M}(t), \tilde{u}_{N, M}(t)\right)+2 \mu b_{N, M}\left(\tilde{u}_{N, M}(t), \tilde{u}_{N, M}(t)\right) \\
& \quad=2 B_{N, M}\left(\tilde{u}_{N, M}(t), \tilde{u}_{N, M}(t), u_{N, M}(t)\right)+2\left(\tilde{f}(t), \tilde{u}_{N, M}(t)\right)_{\omega, N, M} .
\end{aligned}
$$

By Lemma 4.6 and the Hölder inequality,

$$
\begin{aligned}
\mid 2 B_{N, M} & \left(\tilde{u}_{N, M}(t), \tilde{u}_{N, M}(t), u_{N, M}(t)\right) \mid \\
\leq & c\left|u_{N, M}(t)\right|_{1, \omega, N, M, \sim}^{\frac{1}{4}}\left|u_{N, M}(t)\right|_{2, \omega, N, M, \sim}^{\frac{3}{4}}\left|\tilde{u}_{N, M}(t)\right|_{1, \omega, N, M, \sim}^{\frac{1}{4}} \\
& \cdot\left|\tilde{u}_{N, M}(t)\right|_{2, \omega, N, M, \sim}^{\frac{7}{4}} \\
\leq & \mu\left|\tilde{u}_{N, M}(t)\right|_{2, \omega, N, M, \sim}^{2} \\
& +\frac{c}{\mu}\left|u_{N, M}(t)\right|_{1, \omega, N, M, \sim}^{2}\left|u_{N, M}(t)\right|_{2, \omega, N, M, \sim}^{6}\left|\tilde{u}_{N, M}(t)\right|_{1, \omega, N, M, \sim}^{2} .
\end{aligned}
$$

Thanks to Lemma 2.7 and (4.2),

$$
\left|2\left(\tilde{f}(t), \tilde{u}_{N, M}(t)\right)_{\omega, N, M}\right| \leq c\left|\tilde{u}_{N, M}(t)\right|_{1, \omega, N, M, \sim}^{2}+\|\tilde{f}(t)\|_{\omega, N, M}^{2} .
$$

Using (4.22), (4.23) and Lemma 4.3, we get from (4.21) that

$$
\begin{aligned}
& \frac{d}{d t} a_{N, M}\left(\tilde{u}_{N, M}(t), \tilde{u}_{N, M}(t)\right)+\mu\left|\tilde{u}_{N, M}(t)\right|_{2, \omega, N, M, \sim}^{2} \\
& \leq \frac{c}{\mu}\left(\left|u_{N, M}(t)\right|_{1, \omega, N, M, \sim}^{2}\left|u_{N, M}(t)\right|_{2, \omega, N, M, \sim}^{6}+1\right)\left|\tilde{u}_{N, M}(t)\right|_{1, \omega, N, M, \sim}^{2} \\
& \quad+\|\tilde{f}(t)\|_{\omega, N, M}^{2} .
\end{aligned}
$$


Integrating the above inequality with respect to $t$, we get from Lemma 4.1 that

$$
\begin{aligned}
& \left|\tilde{u}_{N, M}(t)\right|_{1, \omega, N, M, \sim}^{2}+\mu \int_{0}^{t}\left|\tilde{u}_{N, M}(\eta)\right|_{2, \omega, N, M, \sim}^{2} d \eta \\
& \quad \leq \frac{c c^{*}}{\mu} \int_{0}^{t}\left|\tilde{u}_{N, M}(\eta)\right|_{1, \omega, N, M, \sim}^{2} d \eta+c \rho\left(\tilde{u}_{0, N, M}, \tilde{f}, t\right) .
\end{aligned}
$$

Finally we use the Gronwall inequality to reach Theorem 3.1.

Next we prove Theorem 3.2. For the sake of simplicity, we first compare the numerical solution $u_{N, M}$ with the orthogonal projection $U_{N, M}=P_{N-1, M-3, q}^{2,0} U, 0<$ $q<1$. Using (2.9) and (2.26), we obtain from (3.4) that

$$
\begin{gathered}
a_{N, M}\left(U_{N, M}(t), \phi\right)+B\left(U_{N, M}(t), U_{N, M}(t), \phi\right)+\mu b_{N, M}\left(U_{N, M}(t), \phi\right) \\
=\sum_{k=1}^{4} G_{k}(\phi)+(f, \phi)_{\omega, N, M}, \quad \forall \phi \in V_{N, M}^{2,0}, t \in(0, T],
\end{gathered}
$$

where

$$
\begin{gathered}
G_{1}(\phi)=\left(\partial_{t} \nabla\left(e^{-\frac{x}{2}}\left(U_{N, M}-U\right)\right), \nabla\left(e^{-\frac{x}{2}} \phi\right)\right), \\
G_{2}(\phi)=B_{N, M}\left(U_{N, M}, U_{N, M}, \phi\right)-B(U, U, \phi), \\
G_{3}(\phi)=\mu\left(\Delta\left(e^{-\frac{x}{2}}\left(U_{N, M}-U\right)\right), \Delta\left(e^{-\frac{x}{2}} \phi\right)\right), \\
G_{4}(\phi)=(f, \phi)_{\omega}-(f, \phi)_{\omega, N, M} .
\end{gathered}
$$

Furthermore, let $\tilde{U}_{N, M}=u_{N, M}-U_{N, M}$. Then we get from (3.7) and (4.3) that

$$
\begin{aligned}
a_{N, M}\left(\tilde{U}_{N, M}(t), \phi\right)+B_{N, M}\left(\tilde{U}_{N, M}(t), U_{N, M}(t)+\tilde{U}_{N, M}(t), \phi\right) & \\
(4.25)+B_{N, M}\left(U_{N, M}(t), \tilde{U}_{N, M}(t), \phi\right)+\mu b_{N, M}\left(\tilde{U}_{N, M}(t), \phi\right) & =-\sum_{j=1}^{4} G_{j}(\phi), \\
\forall \phi & \in V_{N, M}^{2,0}, t \in(0, T] .
\end{aligned}
$$

In addition $\tilde{U}_{N, M}(0)=I_{N, M} U_{0}-P_{N-1, M-3, q}^{2,0} U_{0}$. Comparing (4.25) with (4.20), we find that it suffices to estimate the up-bounds of $\left|G_{j}\left(\tilde{U}_{N, M}(t)\right)\right|$ for determining the accuracy of the numerical solution $u_{N, M}$.

First, we know from (4.13), Lemma 2.10 and result (iii) of Lemma 4.1 that for $r_{1}, s_{1} \geq 2$,

$$
\begin{aligned}
\left|G_{1}\left(\tilde{U}_{N, M}\right)\right| \\
\quad \leq\left\|\nabla\left(e^{-\frac{x}{2}}\left(\partial_{t} U_{N, M}-\partial_{t} U\right)\right)\right\|\left\|\nabla\left(e^{-\frac{x}{2}} \tilde{U}_{N, M}\right)\right\| \\
\quad \leq\left|\partial_{t} U_{N, M}-\partial_{t} U\right|_{1, \omega}\left|\tilde{U}_{N, M}\right|_{1, \omega} \\
\quad \leq \frac{\mu}{8}\left|\tilde{U}_{N, M}\right|_{2, \omega, N, M, \sim}^{2}+\frac{c}{\mu}\left(N^{1-\frac{r_{1}}{2}}+M^{2-s_{1}}\right)^{2}\left(N^{-1}+M^{-2}\right)\left\|\partial_{t} U\right\|_{M_{q}^{r_{1}, s_{1}}}^{2} .
\end{aligned}
$$

Next we have that

$$
\begin{aligned}
\left|G_{2}\left(\tilde{U}_{N, M}\right)\right| \leq & \left|B\left(U, U, \tilde{U}_{N, M}\right)-B_{N, M}\left(U, U, \tilde{U}_{N, M}\right)\right| \\
& \quad+\left|B_{N, M}\left(U, U, \tilde{U}_{N, M}\right)-B_{N, M}\left(U_{N, M}, U_{N, M}, \tilde{U}_{N, M}\right)\right| \\
\leq & \sum_{j=1}^{5} A_{j},
\end{aligned}
$$


where

$$
\begin{aligned}
& A_{1}= \mid\left(e^{\frac{x}{2}} \Delta\left(e^{-\frac{x}{2}} U\right) \partial_{y}\left(e^{-\frac{x}{2}} U\right), e^{\frac{x}{2}} \partial_{x}\left(e^{-\frac{x}{2}} \tilde{U}_{N, M}\right)\right)_{\omega} \\
&-\left(e^{\frac{x}{2}} \Delta\left(e^{-\frac{x}{2}} U\right) \partial_{y}\left(e^{-\frac{x}{2}} U\right), e^{\frac{x}{2}} \partial_{x}\left(e^{-\frac{x}{2}} \tilde{U}_{N, M}\right)\right)_{\omega, N, M} \mid, \\
& A_{2}= \mid\left(e^{\frac{x}{2}} \Delta\left(e^{-\frac{x}{2}} U\right) \partial_{x}\left(e^{-\frac{x}{2}} U\right), e^{\frac{x}{2}} \partial_{y}\left(e^{-\frac{x}{2}} \tilde{U}_{N, M}\right)\right)_{\omega} \\
&-\left(e^{\frac{x}{2}} \Delta\left(e^{-\frac{x}{2}} U\right) \partial_{x}\left(e^{-\frac{x}{2}} U\right), e^{\frac{x}{2}} \partial_{y}\left(e^{-\frac{x}{2}} \tilde{U}_{N, M}\right)\right)_{\omega, N, M} \mid, \\
& A_{3}= \mid\left(e^{\frac{x}{2}} \Delta\left(e^{-\frac{x}{2}}\left(U-e_{N, M}\right)\right), \partial_{y} U_{N, M} \partial_{x}\left(e^{-\frac{x}{2}} \tilde{U}_{N, M}\right)\right. \\
&\left.\left.A_{4}\right) \partial_{y} \tilde{U}_{N, M}\right)_{\omega, N, M} \mid, \\
& A_{5}=\left|\left(e^{\frac{x}{2}} \Delta\left(e^{-\frac{x}{2}} U\right), \partial_{y}\left(U-U_{N, M}\right) \partial_{x}\left(e^{-\frac{x}{2}} \tilde{U}_{N, M}\right)\right)_{\omega, N, M}\right|, \\
&
\end{aligned}
$$

We now estimate $A_{i}$. We have from imbedding theory that for any $v \in H_{\omega}^{1+\delta}(\Omega)$ and $\delta>0$,

$$
\left\|e^{-\frac{x}{2}} v\right\|_{L^{\infty}(\Omega)} \leq c\left\|e^{-\frac{x}{2}} v\right\|_{1+\delta} \leq c\|v\|_{1+\delta, \omega}
$$

Obviously

$$
\partial_{x}^{r}\left(\partial_{x}^{2}\left(e^{-\frac{x}{2}} U\right) \partial_{y} U\right)=\sum_{j=0}^{r}\left(\begin{array}{c}
r \\
j
\end{array}\right) \partial_{x}^{j+2}\left(e^{-\frac{x}{2}} U\right) \partial_{x}^{r-j} \partial_{y} U .
$$

Hence by (4.28),

$$
\begin{aligned}
& \left\|\partial_{x}^{2}\left(e^{-\frac{x}{2}} U\right) \partial_{y} U\right\|_{L^{2}\left(\Lambda_{2}, H_{\omega q}^{r}\left(\Lambda_{1}\right)\right)} \\
& \quad \leq\|U\|_{\left[\frac{r+1}{2}\right]+2+\delta, \omega}\|U\|_{L^{2}\left(\Lambda_{2}, H_{\omega q}^{r+2}\left(\Lambda_{1}\right)\right) \cap H^{1}\left(\Lambda_{2}, H_{\omega q}^{r}\left(\Lambda_{1}\right)\right)} .
\end{aligned}
$$

Similarly

$$
\begin{aligned}
& \left\|\partial_{y}^{2}\left(e^{-\frac{x}{2}} U\right) \partial_{y} U\right\|_{L^{2}\left(\Lambda_{2}, H_{\omega q}^{r}\left(\Lambda_{1}\right)\right)} \\
& \left.\quad \leq c\|U\|_{\left[\frac{r+1}{2}\right]+2+\delta, \omega}\|U\|_{H^{2}\left(\Lambda_{2}, H_{\omega}^{r}\left(\Lambda_{1}\right)\right) \cap H^{1}\left(\Lambda_{2}, H_{\omega}^{r} q\right.}^{r}\left(\Lambda_{1}\right)\right), \\
& \quad\left\|\partial_{x}^{2}\left(e^{-\frac{x}{2}} U\right) \partial_{y} U\right\|_{H^{s}\left(\Lambda_{2}, L_{\omega}^{2}\left(\Lambda_{1}\right)\right)} \\
& \quad \leq c\|U\|_{\left[\frac{s+1}{2}\right]+2+\delta, \omega}\|U\|_{H^{s}\left(\Lambda_{2}, H_{\omega}^{2} q\left(\Lambda_{1}\right)\right) \cap H^{s+1}\left(\Lambda_{2}, L_{\omega}^{2} q\left(\Lambda_{1}\right)\right)}, \\
& \quad\left\|\partial_{y}^{2}\left(e^{-\frac{x}{2}} U\right) \partial_{y} U\right\|_{H^{s}\left(\Lambda_{2}, L_{\omega q}^{2}\left(\Lambda_{1}\right)\right)} \\
& \quad \leq c\|U\|_{\left[\frac{s+1}{2}\right]+2+\delta, \omega}\|U\|_{H^{s+2}\left(\Lambda_{2}, L_{\omega}^{2} q\left(\Lambda_{1}\right)\right) \cap H^{s+1}\left(\Lambda_{2}, L_{\omega q}^{2}\left(\Lambda_{1}\right)\right)} .
\end{aligned}
$$

Therefore we use Lemma 2.11, Theorem 2.2 and result (iii) of Lemma 4.1 to obtain that

$$
\begin{aligned}
A_{1} & \leq c\left(N^{-\frac{r}{2}}\|U\|_{Y_{q, \delta}^{r, s}}^{2}+M^{-s}\|U\|_{Y_{q, \delta}^{r, s}}^{2}\left|\tilde{U}_{N, M}\right|_{1, \omega}\right) \\
& \leq \frac{\mu}{40}\left|\tilde{U}_{N, M}\right|_{2, \omega, N, M, \sim}^{2}+\frac{c}{\mu}\left(N^{-\frac{\tilde{r}}{2}}+M^{-\tilde{s}}\right)^{2}\|U\|_{\tilde{Y}_{q, \delta}^{r, s}}^{4} .
\end{aligned}
$$


We can derive an up-bound of $A_{2}$, which is exactly the same as (4.29). Clearly by (2.29),

$$
\begin{aligned}
A_{3} \leq & \left\|e^{\frac{x}{2}} \Delta\left(e^{-\frac{x}{2}}\left(U-U_{N, M}\right)\right)\right\|_{\omega, N, M} \\
& \cdot\left\|\partial_{y} U_{N, M} \partial_{x}\left(e^{-\frac{x}{2}} \tilde{U}_{N, M}\right)-\partial_{x}\left(e^{-\frac{x}{2}} U_{N, M}\right) \partial_{y}\left(e^{-\frac{x}{2}} \tilde{U}_{N, M}\right)\right\|_{\omega, N, M} .
\end{aligned}
$$

Next we estimate $\left\|e^{\frac{x}{2}} \Delta\left(e^{-\frac{x}{2}}\left(U-U_{N, M}\right)\right)\right\|_{\omega, N, M}$. By $(2.32)$, we have that

$$
\begin{aligned}
\| e^{\frac{x}{2}} \Delta & \left(e^{-\frac{x}{2}}\left(U-U_{N, M}\right)\right) \|_{\omega, N, M} \\
\leq & \left\|I_{N, M}\left(e^{\frac{x}{2}} \Delta\left(e^{-\frac{x}{2}} U\right)\right)-I_{N-1, M-2}\left(e^{\frac{x}{2}} \Delta\left(e^{-\frac{x}{2}} U\right)\right)\right\|_{\omega, N, M} \\
& +\left\|I_{N-1, M-2}\left(e^{\frac{x}{2}} \Delta\left(e^{-\frac{x}{2}} U\right)\right)-e^{\frac{x}{2}} \Delta\left(e^{-\frac{x}{2}} U_{N, M}\right)\right\|_{\omega, N, M} .
\end{aligned}
$$

It is easy to see that

$$
I_{N, M}\left(e^{\frac{x}{2}} \Delta\left(e^{-\frac{x}{2}} U\right)\right)-I_{N-1, M-2}\left(e^{\frac{x}{2}} \Delta\left(e^{-\frac{x}{2}} U\right)\right) \in V_{N, M}^{2,0}
$$

and

$$
I_{N-1, M-2}\left(e^{\frac{x}{2}} \Delta\left(e^{-\frac{x}{2}} U\right)\right)-e^{\frac{x}{2}} \Delta\left(e^{-\frac{x}{2}} U_{N, M}\right) \in V_{N-1, M-2} .
$$

Thus by (2.31), Remark 2.6 and (4.30), we obtain that

$$
\begin{aligned}
&\left\|e^{\frac{x}{2}} \Delta\left(e^{-\frac{x}{2}}\left(U-U_{N, M}\right)\right)\right\|_{\omega, N, M} \\
& \leq c\left\|e^{\frac{x}{2}} \Delta\left(e^{-\frac{x}{2}} U\right)-I_{N, M}\left(e^{\frac{x}{2}} \Delta\left(e^{-\frac{x}{2}} U\right)\right)\right\|_{\omega} \\
&+c\left\|e^{\frac{x}{2}} \Delta\left(e^{-\frac{x}{2}} U\right)-I_{N-1, M-2}\left(e^{\frac{x}{2}} \Delta\left(e^{-\frac{x}{2}} U\right)\right)\right\|_{\omega} \\
&+c\left\|e^{\frac{x}{2}} \Delta\left(e^{-\frac{x}{2}} U\right)-e^{\frac{x}{2}} \Delta\left(e^{-\frac{x}{2}} U_{N, M}\right)\right\|_{\omega} .
\end{aligned}
$$

By Theorem 2.2,

$$
\begin{aligned}
\| e^{\frac{x}{2}} \Delta & \left(e^{-\frac{x}{2}} U\right)-I_{N, M}\left(e^{\frac{x}{2}} \Delta\left(e^{-\frac{x}{2}} U\right)\right) \|_{\omega} \\
\leq & c\left(\left\|\partial_{x}^{2} U-I_{N, M} \partial_{x}^{2} U\right\|_{\omega}+\left\|\partial_{x} U-I_{N, M} \partial_{x} U\right\|_{\omega}\right. \\
& \left.+\left\|U-I_{N, M} U\right\|_{\omega}+\left\|\partial_{y}^{2} U-I_{N, M} \partial_{y}^{2} U\right\|_{\omega}\right) \\
\leq & c\left(N^{2-\frac{r}{2}}\|U\|_{L^{2}\left(\Lambda_{2}, H_{\omega}^{r} q\left(\Lambda_{1}\right)\right)}+M^{2-s}\|U\|_{H^{s}\left(\Lambda_{2}, L_{\omega}^{2}\left(\Lambda_{1}\right)\right)}\right) .
\end{aligned}
$$

By Lemma 2.10,

$$
\left\|e^{\frac{x}{2}} \Delta\left(e^{-\frac{x}{2}} U\right)-e^{\frac{x}{2}} \Delta\left(e^{-\frac{x}{2}} U_{N, M}\right)\right\|_{\omega} \leq c\left(N^{1-\frac{r}{2}}+M^{2-s}\right)\|U\|_{M_{q}^{r, s} .}
$$

The above statements lead to that for any $0<q_{1}<q<1$,

$$
\left\|e^{\frac{x}{2}} \Delta\left(e^{-\frac{x}{2}}\left(U-U_{N, M}\right)\right)\right\|_{\omega, N, M} \leq c\left(N^{2-\frac{r}{2}}+M^{2-s}\right)\|U\|_{\tilde{M}_{q_{1}}^{r, s}} .
$$

Since $U_{N, M}, \tilde{U}_{N, M} \in V_{N, M}^{2,0}(\Omega)$, we obtain from the Schwartz inequality and Lemma 4.4 that

$$
\begin{aligned}
& \left\|\partial_{y} U_{N, M} \partial_{x}\left(e^{-\frac{x}{2}} \tilde{U}_{N, M}\right)-\partial_{x}\left(e^{-\frac{x}{2}} U_{N, M}\right) \partial_{y} \tilde{U}_{N, M}\right\|_{\omega, N, M} \\
& \quad \leq c\left(\left|U_{N, M}\right|_{1, \omega, N, M, \sim}\left|\tilde{U}_{N, M}\right|_{1, \omega, N, M, \sim}\right)^{\frac{1}{4}}\left(\left|U_{N, M}\right|_{2, \omega, N, M, \sim}\left|\tilde{U}_{N, M}\right|_{2, \omega, N, M, \sim}\right)^{\frac{3}{4}} .
\end{aligned}
$$

By Lemma 4.1 and Lemma 2.10, we have

$$
\begin{aligned}
& \left|U_{N, M}\right|_{1, \omega, N, M, \sim} \leq c\left|U_{N, M}\right|_{1, \omega} \leq c\|U\|_{M_{q}^{2,2}}, \\
& \left|U_{N, M}\right|_{2, \omega, N, M, \sim} \leq c\left|U_{N, M}\right|_{2, \omega} \leq c\|U\|_{M_{q}^{2,2} .}
\end{aligned}
$$


The above statements lead to

$$
\begin{aligned}
A_{3} \leq & c\left(N^{2-\frac{r}{2}}+M^{2-s}\right)\|U\|_{\tilde{M}_{q}^{r, s}}\|U\|_{M_{q}^{2,2}} \\
& \cdot\left(\left|\tilde{U}_{N, M}\right|_{1, \omega, N, M, \sim}\right)^{\frac{1}{4}}\left(\left|\tilde{U}_{N, M}\right|_{2, \omega, N, M, \sim}\right)^{\frac{3}{4}} \\
\leq & c\left(N^{2-\frac{r}{2}}+M^{2-s}\right)^{2}\|U\|_{\tilde{M}_{q 1}^{r, s}}^{2}\|U\|_{M_{q 1}^{2,2}}^{2} \\
& +\frac{c}{\mu}\left|\tilde{U}_{N, M}\right|_{1, \omega, N, M, \sim}^{2}+\frac{\mu}{40}\left|\tilde{U}_{N, M}\right|_{2, \omega, N, M, \sim}^{2}
\end{aligned}
$$

We now estimate $A_{4}$. By the Schwartz inequality,

$$
A_{4} \leq\left\|\partial_{y}\left(U-U_{N, M}\right)\right\|_{\omega, N, M}\left\|\Delta\left(e^{-\frac{x}{2}} U\right)\right\|_{L^{\infty}(\Omega)}\left\|e^{\frac{x}{2}} \partial_{x}\left(e^{-\frac{x}{2}} \tilde{U}_{N, M}\right)\right\|_{\omega, N, M} .
$$

Since $I_{N, M}\left(\partial_{y} U\right)-I_{N, M-1}\left(\partial_{y} U\right) \in V_{N, M}^{2,0}, I_{N, M-1}\left(\partial_{y} U\right)-\partial_{y} U_{N, M} \in V_{N, M-1}^{1,0}$, we obtain from (2.31), Lemma 2.4, Remark 2.4, Theorem 2.2 and Lemma 2.10 that

$$
\begin{aligned}
&\left\|\partial_{y}\left(U-U_{N, M}\right)\right\|_{\omega, N, M} \\
& \leq\left\|I_{N, M}\left(\partial_{y} U\right)-I_{N, M-1}\left(\partial_{y} U\right)\right\|_{\omega, N, M}+\left\|\partial_{y} U_{N, M}-I_{N, M-1}\left(\partial_{y} U\right)\right\|_{\omega, N, M} \\
& \leq c\left\|\partial_{y} U-I_{N, M-1}\left(\partial_{y} U\right)\right\|_{\omega}+c\left\|\partial_{y} U-I_{N, M}\left(\partial_{y} U\right)\right\|_{\omega} \\
& \quad+c\left\|\partial_{y} U-\partial_{y} P_{N-1, M-3}^{2,0} U\right\|_{\omega} \\
& \leq c\left(N^{\frac{3-r}{2}}\left\|\partial_{y} U\right\|_{L^{2}\left(\Lambda_{2}, H_{\omega q}^{r-1}\left(\Lambda_{1}\right)\right)}+M^{1-s}\left\|\partial_{y} U\right\|_{H^{s-1}\left(\Lambda_{2}, L_{\omega}^{2} q\right.}\left(\Lambda_{1}\right)\right) \\
&\left.\quad+\left(N^{1-\frac{r}{2}}+M^{2-s}\right)\left(N^{-1}+M^{-2}\right)^{\frac{1}{2}}\|U\|_{M_{q}^{r, s}}\right) .
\end{aligned}
$$

By Lemma 2.4 and Remark 2.2,

$$
\left\|e^{\frac{x}{2}} \partial_{x}\left(e^{-\frac{x}{2}} \tilde{U}_{N, M}\right)\right\|_{\omega, N, M} \leq\left|\tilde{U}_{N, M}\right|_{1, \omega, N, M, \sim}
$$

By imbedding theory, for $\delta>0$,

$$
\left\|\Delta\left(e^{-\frac{x}{2}} U\right)\right\|_{L^{\infty}(\Omega)} \leq c\left\|e^{\frac{x}{2}} \Delta\left(e^{-\frac{x}{2}} U\right)\right\|_{H_{\omega}^{1+\delta}(\Omega)} \leq c\|U\|_{H^{3+\delta}(\Omega)} .
$$

The above statements lead to

$$
\begin{aligned}
& A_{4} \leq c\left(\left(N^{\frac{3-r}{2}}+M^{1-s}\right)^{2}+\left(N^{\frac{4-r}{2}}+M^{2-s}\right)^{2}\left(N^{-1}+M^{-2}\right)\right)\|U\|_{\tilde{M}_{q_{1}}^{r, s}}^{2}\|U\|_{H^{3+\delta}(\Omega)}^{2} \\
& \quad+\left|\tilde{U}_{N, M}\right|_{1, \omega, N, M, \sim}^{2} .
\end{aligned}
$$

By the same procedure as in the derivation of (4.31), we can get the same error as (4.31) for $A_{5}$.

According to (4.14) and Lemmas 2.10 and 4.1, we assert that for $0<q<1$ and $r, s \geq 2$,

$$
\begin{aligned}
\left|G_{3}\left(\tilde{U}_{N, M}\right)\right| & \leq \mu\left\|\Delta\left(e^{-\frac{x}{2}}\left(U-U_{N, M}\right)\right)\right\|\left\|\Delta\left(e^{-\frac{x}{2}} \tilde{U}_{N, M}\right)\right\| \\
& \leq \mu\left|U-U_{N, M}\right|_{2, \omega}\left|\tilde{U}_{N, M}\right|_{2, \omega} \\
& \leq \frac{\mu}{8}\left|\tilde{U}_{N, M}\right|_{2, \omega, N, M, \sim}^{2}+c\left(N^{1-\frac{r}{2}}+M^{2-s}\right)^{2} \max (N, M)\|U\|_{M_{q}^{r, s}}^{2} .
\end{aligned}
$$


We know from Lemmas 2.11 and 4.1 and Theorem 3.2 that for $0<q<1$ and $\lambda \geq 1, \gamma>\frac{3}{2}$,

$$
\begin{aligned}
\left|G_{4}\left(\tilde{U}_{N, M}\right)\right| \leq & c\left(\left\|f-I_{N-1, M-3} f\right\|_{\omega}+\left\|f-I_{N, M} f\right\|_{\omega}\right)\left\|\tilde{U}_{N, M}\right\|_{\omega} \\
\leq & \frac{\mu}{8}\left|\tilde{U}_{N, M}\right|_{2, \omega, N, M, \sim}^{2} \\
& \quad+\frac{c}{\mu}\left(N^{-\frac{\lambda}{2}}\|f\|_{L^{2}\left(\Lambda_{2}, H_{\omega q}^{\lambda}\left(\Lambda_{1}\right)\right)}+M^{-\gamma}\|f\|_{H^{\gamma}\left(\Lambda_{2}, L_{\omega q}^{2}\left(\Lambda_{1}\right)\right)}\right)^{2} .
\end{aligned}
$$

We now estimate the initial error. By Lemma 4.1, Lemma 2.10 and Theorem 2.2, we get that

$$
\begin{aligned}
& a_{N, M}\left(\tilde{U}_{N, M}(0), \tilde{U}_{N, M}(0)\right) \\
& \leq c\left|U_{0}-P_{N-1, M-3, q}^{2,0} U_{0}\right|_{1, \omega}^{2}+c\left|U_{0}-I_{N, M} U_{0}\right|_{1, \omega}^{2} \\
&(4.35) \leq c\left(N^{1-\frac{r_{0}}{2}}+M^{2-s_{0}}\right)^{2}\left(N^{-1}+M^{-2}\right)\left\|U_{0}\right\|_{M_{q}^{r_{0}, s_{0}}}^{2} \\
&+c\left(N^{2-\frac{r_{0}}{2}}\left\|U_{0}\right\|_{L^{2}\left(\Lambda_{2}, H_{\omega q}^{r_{0}}\left(\Lambda_{1}\right)\right)}+M^{1-s_{0}}\left\|U_{0}\right\|_{H^{s_{0}}\left(\Lambda_{2}, L_{\omega}^{2} q\left(\Lambda_{1}\right)\right)}\right)^{2} \\
& \leq c\left(\left(N^{2-\frac{r_{0}}{2}}+M^{1-s_{0}}\right)^{2}+\left(N^{1-\frac{r_{0}}{2}}+M^{2-s_{0}}\right)^{2}\left(N^{-1}+M^{-2}\right)\right)\left\|U_{0}\right\|_{\tilde{M}_{q_{1}}^{r_{0}, s_{0}}}^{2} .
\end{aligned}
$$

Also the combination of Lemma 4.1 and Lemma 2.10 implies that

$$
c^{*}\left(U_{N, M}\right) \leq\|U\|_{C\left(0, T ; H_{\omega q}^{2}(\Omega)\right)}^{8}+1 .
$$

Finally we use (4.26)-(4.27), (4.29)-(4.35) to finish the proof of Theorem 3.2.

\section{Some CONCLUding REMARKS}

In this paper, we consider the stream function form of incompressible fluid flow, and so we keep the physical boundary condition in actual computation. This avoids the numerical boundary layer caused by the nonphysical boundary condition and makes the calculation stable.

We use the Laguerre-Legendre pseudospectral approximation. So unlike the Galerkin method, we do not use some quadratures on unbounded domains for calculating the Laguerre coefficients, which is costly and usually not accurate.

We use the transformation $W={ }^{-\frac{x}{2}} U$ so that the resulting system is well posed in the weighted space and possesses the conservations as in the continuous case. If we use the discrete inner product with uniform weight for the transformed function, then the problem is not well posed and does not preserve the conservations. We may not use this transformation and take the functions $e^{-\frac{x}{2}} \mathcal{L}_{l}(x)$ as the base functions. But in this case, we need to build up a set of results for the spectral approximation by using the system $\left\{e^{-\frac{x}{2}} \mathcal{L}_{l}(x)\right\}$. This is not easy for spectral approximation of partial differential equations of fourth order.

The method in this paper can be used for several kinds of problems. For instance, we consider the fluid flow in a very long river that is walled by a dam. When the water follows from the gate of the dam, we need to consider the fluid flow in a strip that is walled at one end. Another important motivation of this work is the numerical simulation of exterior problems. For example, if the obstacle is rectangular, then we may divide the whole domain into eight subdomains (see Figure 3 ). In this case, we could use the two-dimensional Laguerre approximation on the subdomains $D_{1}, D_{3}, D_{5}$ and $D_{7}$, while we should use Laguerre-Legendre approximation on the 


\begin{tabular}{c|c|c}
$D_{1}$ & $D_{8}$ & $D_{7}$ \\
\hline$D_{2}$ & obstacle & $D_{6}$ \\
\hline$D_{3}$ & $D_{4}$ & $D_{5}$
\end{tabular}

FiguRE 3.

subdomains $D_{2}, D_{4}, D_{6}$ and $D_{8}$. If the obstacle is not rectangular, then we can use the finite element method coupled with the method in this paper.

The main disadvantage of scheme (3.7) is that we need to solve a Laplace equation numerically at each time step. This feature comes from using the pseudospectral method for the high order differential equation (1.1). But if we solve the primitive form of the Navier-Stokes equation, then we need the nonphysical boundary condition on the pressure. On the other hand, if we solve the vorticity-stream form of the Navier-stokes equation, then we need the nonphysical boundary condition on the vorticity. They usually induce serious numerical errors. We may use the Galerkin method and take some special functions as base functions for solving (1.1) so that the corresponding matrix might not be full. But in this case, we have to use some quadratures on the unbounded domain to calculate the Laguerre coefficients. This is also costly and not as accurate as we usually expect. An interesting open problem is: Can we find a pseudospectral method for the Laplace equation on the unbouded domain so that the corresponding matrix is not full?

\section{Appendix A}

We have from David and Rabinowitz (see page 39 of 7 ) that

$$
\mathcal{L}_{N+1}(x)=\frac{(-1)^{N+1}}{(N+1) !} x^{N+1}+\cdots+\alpha_{1} x^{3}+\beta_{1} x^{2}+\gamma_{1} x+\tau_{1},
$$

where

$$
\alpha_{1}=-\frac{1}{36} N\left(N^{2}-1\right), \quad \beta_{1}=\frac{1}{4} N(N+1), \quad \gamma_{1}=-(N+1), \quad \tau_{1}=1 .
$$

Consequently

$$
\begin{aligned}
\mathcal{L}_{N+1}(0) & =1, \\
\partial_{x} \mathcal{L}_{N+1}(0) & =-(N+1), \\
\partial_{x}^{2} \mathcal{L}_{N+1}(0) & =\frac{1}{2} N(N+1), \\
\partial_{x}^{3} \mathcal{L}_{N+1}(0) & =-\frac{1}{6} N\left(N^{2}-1\right) .
\end{aligned}
$$

By integration by parts, (A.2) and the orthogonality (2.3) with $\alpha=0$, we get that

$$
\begin{aligned}
\int_{\Lambda_{1}} \partial_{x}^{2} \mathcal{L}_{N+1}(x) e^{-x} d x & =\left.e^{-x} \partial_{x} \mathcal{L}_{N+1}(x)\right|_{0} ^{\infty}+\int_{\Lambda_{1}} \partial_{x} \mathcal{L}_{N+1}(x) e^{-x} d x \\
& =\left.e^{-x} \partial_{x} \mathcal{L}_{N+1}(x)\right|_{0} ^{\infty}+\left.e^{-x} \mathcal{L}_{N+1}(x)\right|_{0} ^{\infty}=N
\end{aligned}
$$

Similarly,

$$
\begin{aligned}
\int_{\Lambda_{1}} \partial_{x}^{2} \mathcal{L}_{N+1}(x) x e^{-x} d x & =-\int_{\Lambda_{1}} \partial_{x} \mathcal{L}_{N+1}(x) \partial_{x}\left(x e^{-x}\right) d x \\
& =-\left.\partial_{x}\left(x e^{-x}\right) \mathcal{L}_{N+1}(x)\right|_{0} ^{\infty}+\int_{\Lambda_{1}} \mathcal{L}_{N+1}(x) \partial_{x}^{2}\left(x e^{-x}\right) d x=1 .
\end{aligned}
$$


Since the $\sigma_{j}^{N}$ are the zeros of the equation $\partial_{x}^{2} \mathcal{L}_{N+1}(x)=0$, we get from (A.1) that

$$
\partial_{x}^{2} \mathcal{L}_{N+1}(x)=\frac{(-1)^{N+1}}{(N-1) !}\left(x-\sigma_{1}^{N}\right) \cdots\left(x-\sigma_{N-1}^{N}\right)=\frac{(-1)^{N+1}}{(N-1) !} X(x) .
$$

Letting $x=0$ in the above equation, we obtain that

$$
\sigma_{1}^{N} \cdots \sigma_{N-1}^{N}=(N-1) ! \partial_{x}^{2} \mathcal{L}_{N+1}(0)
$$

Differentiating both sides of (A.5) yields that

$$
\sum_{j=1}^{N-1} \sigma_{1}^{N} \cdots \sigma_{j-1}^{N} \sigma_{j+1}^{N} \cdots \sigma_{N-1}^{N}=-(N-1) ! \partial_{x}^{3} \mathcal{L}_{N+1}(0) .
$$

Substituting (A.5) and (A.6) into the definition of $\xi_{1}^{N}$, we get from (A.2) and (A.4) that

$$
\xi_{1}^{N}=\int_{\Lambda_{1}} \partial_{x}^{2} \mathcal{L}_{N+1}(x) x e^{-x} d x\left(\partial_{x}^{2} \mathcal{L}_{N+1}(0)\right)^{-1}=\frac{2}{N(N+1)} .
$$

Similarly, substituting (A.5)-(A.7) into the definition of $\xi_{0}^{N}$, we get from (A.2) and (A.3) that

$$
\begin{gathered}
\xi_{0}^{N}=\left(\int_{\Lambda_{1}} \partial_{x}^{2} \mathcal{L}_{N+1}(x) e^{-x} d x-\partial_{x}^{3} \mathcal{L}_{N+1}(0) \xi_{1}^{N}\right)\left(\partial_{x}^{2} \mathcal{L}_{N+1}(0)\right)^{-1} \\
=\frac{6 N+N\left(N^{2}-1\right) \xi_{1}^{N}}{3 N(N+1)}=\frac{2(4 N-1)}{3 N(N+1)}
\end{gathered}
$$

\section{APPENDiX B}

We first prove that for any $0<\alpha<1$,

$$
\sum_{j=1}^{N-1} e^{(1-\alpha) \sigma_{j}^{N}} \omega_{j}^{N} \leq e+\frac{2}{\alpha^{2}}
$$

Indeed, by (3.6.5) of Davis and Rabinowitz [7, we have that (B.2)

$$
\int_{0}^{\infty} f(x) x^{2} e^{-x} d x=\sum_{j=1}^{N-1} f\left(\sigma_{j}^{N}\right) \tilde{\omega}_{j}^{N}+\frac{(N-1) !(N+1) !}{(2 N-2) !} f^{(2 N-2)}(\xi), \quad 0<\xi<\infty .
$$

Let $f(x)=e^{(1-\alpha) x}$ in (B.2). Then

$$
\begin{aligned}
\int_{0}^{\infty} x^{2} e^{-\alpha x} d x= & \sum_{j=1}^{N-1} e^{(1-\alpha) \sigma_{j}^{N}} \tilde{\omega}_{j}^{N}+\frac{(N-1) !(N+1) !}{(2 N-2) !}(1-\alpha)^{2 N-2} e^{(1-\alpha) \xi_{1}} \\
& \geq \sum_{j=1}^{N-1} e^{(1-\alpha) \sigma_{j}^{N}} \tilde{\omega}_{j}^{N}
\end{aligned}
$$

and so

$$
\sum_{j=1}^{N-1} e^{(1-\alpha) \sigma_{j}^{N}} \tilde{\omega}_{j}^{N} \leq \frac{2}{\alpha^{2}}
$$


Because $\tilde{\omega}_{j}^{N}=\left(\sigma_{j}^{N}\right)^{2} \omega_{j}^{N}$, we have from (B.3) that

$$
\sum_{\sigma_{j}^{N} \geq 1} e^{(1-\alpha) \sigma_{j}^{N}} \omega_{j}^{N} \leq \sum_{\sigma_{j}^{N} \geq 1} e^{(1-\alpha) \sigma_{j}^{N}} \tilde{\omega}_{j}^{N} \leq \frac{2}{\alpha^{2}} .
$$

On the other hand, we take $f(x)=\frac{1}{x^{2}}$ in (B.2) to get that

$$
\begin{aligned}
1 & =\sum_{j=1}^{N-1} \omega_{j}^{N}+\frac{(2 N-1)(N-1) !(N+1) !}{\xi_{2}^{2 N}} \geq \sum_{j=1}^{N-1} \omega_{i}^{N} \geq \sum_{\sigma_{j}^{N}<1} \omega_{j}^{N} \\
& \geq e^{-1} \sum_{\sigma_{j}^{N}<1} e^{(1-\alpha) \sigma_{j}^{N}} \omega_{j}^{N} .
\end{aligned}
$$

The combination of (B.4) and (B.5) leads to the desired result.

We now prove (2.20). In fact,

$$
\begin{aligned}
\|v\|_{\omega, N, \Lambda_{1}}^{2} & =\sum_{j=1}^{N-1} v^{2}\left(\sigma_{j}^{N}\right) \omega_{j}^{N}=\sum_{j=1}^{N-1} v^{2}\left(\sigma_{j}^{N}\right) e^{-(1-\alpha) \sigma_{j}^{N}}\left(\omega_{j}^{N} e^{(1-\alpha) \sigma_{j}^{N}}\right) \\
& \leq\left\|v e^{-\frac{1-\alpha}{2} x}\right\|_{L^{\infty}\left(\Lambda_{1}\right)}^{2} \sum_{j=1}^{N-1} \omega_{j}^{N} e^{(1-\alpha) \sigma_{j}^{N}} .
\end{aligned}
$$

Moreover (see Maday, Pernaud-Thomas and Vandeven [17]), the space

$$
\left[H_{\omega^{1-\alpha}}^{\frac{1}{2}+\epsilon}\left(\Lambda_{1}\right), H_{\omega^{1-\alpha}}^{\frac{1}{2}-\epsilon}\left(\Lambda_{1}\right)\right]_{\frac{1}{2}} \subset L_{\omega^{1-\alpha}}^{\infty}\left(\Lambda_{1}\right) .
$$

Hence

$$
\left\|v e^{-\frac{1-\alpha}{2} x}\right\|_{L^{\infty}\left(\Lambda_{1}\right)}^{2} \leq c\|v\|_{H_{\omega^{1-\alpha}}^{\frac{1}{2}+\epsilon}\left(\Lambda_{1}\right)}\|v\|_{H_{\omega^{1-\alpha}}^{\frac{1}{2}-\epsilon}\left(\Lambda_{1}\right)} .
$$

The combination of (B.1), (B.6) and (B.7) completes the proof.

\section{REFERENCES}

[1] R. A. Adams, Sobolev Spaces, Academic Press, New York, 1975. MR 56:9247

[2] C. Bernardi, G. Coppoletta and Y. Maday, Some spectral approximations of multidimensional fourth-order problems, Internal Report 90021, Laboratoire $d$ ' Analyse Numérique, Université Pierre et Marie Curie, Paris, 1990.

[3] C. Bernardi and Y. Maday, Basic Results on Spectral Methods, R94022, Univ. Pierre et Marie Curie, Paris, 1994

[4] C. Bernardi and Y. Maday, Spectral methods, in Handbook of Numerical Analysis, 209-486, ed. by P. G. Ciarlet and J. L. Lions, Elsevier, Amsterdam, 1997.

[5] J. P. Boyd, Spectral methods using rational basis function in an infinite interval, J. Comp. Phys., 69(1987), 112-142. MR 88e:65093

[6] O. Coulaud, D. Funaro and O.Kavian, Laguerre spectral approximation of elliptic problems in exterior domains, Comp. Mech. in Appl. Mech and Engi., 80 (1990), 451-458.

[7] P.J. Davis and P. Rabinowitz, Methods of Numerical Integration, Academic Press, New York, 1984. MR 86d:65004

[8] D. Funaro and O. Kavian, Approximation of some diffusion evolution equations in unbounded domains by Hermite functions, Math. Comp., 57(1990), 597-619. MR 92k:35156

[9] Guo Ben-yu, Error estimation for Hermite spectral method for nonlinear partial differential equations, Math. Comp., 68(1999), 1067-1078. MR 99i:65111

[10] Guo Ben-yu and He Li-ping, The fully discrete Legendre spectral approximation of twodimensional unsteady incompressible fluid flow in steam function form, SIAM J. Numer. Anal., 35(1998), 146-176. MR 99f:76103 
[11] Guo Ben-yu and Jie Shen, Laguerre-Galerkin method for nonlinear partial differential equations on a semi-infinite interval, Numer. Math., 86(2000), 635-654. MR 2001:65152

[12] Guo Ben-yu and Xu Cheng-long, Hermite pseudospectral method for nonlinear partial differential equations, RAIRO Math. Model and Numer. Anal., 34(2000), 859-872. MR 2001f: 65142

[13] Guo Ben-yu and Xu Cheng-long, On two-dimensional unsteady incompressible fluid flow in an infinity strip, Math. Meth. in Appl. Sci., 23(2000), 1617-1636. MR 2001j:76030

[14] J. R. Kweon and R. B. Kellogg, Smooth solution of the compressible Navier-Stokes equations in an unbounded domain with inflow boundary condition, J. Math. Anal. Appl., 220(1998), 657-675. MR 99b:35169

[15] J. L. Lions, Quèlques méthodes de Résolution des Problèms aux Limités Non Linéaires, Dunod, Paris, 1969. MR 41:4326

[16] J. L. Lions and E. Magenes, Nonhomogeneous boundary value problems and applications, Vol I, Dunod, Paris, 1972. MR 50:2670

[17] Y. Maday, B. Pernaud-Thomas and H. Vandeven, One réhabilitation des méthods spèctrales de type Laguerre, Rech. Aérospat., 6(1985), 353-379. MR 88b:65135

[18] G. Mastroianni and G. Monegato, Nyström interpolants based on zeros of Laguerre polynomials for some Weiner-Hopf equations, IMA J. of Numer. Anal., 17(1997), 621-642. MR 98j:45011

[19] G.Szegö, Orthogonal Polynomials, Amer. Math, Soc., New York, 1959. MR 21:5029

[20] R. Temam, Navier-Stokes Equation, North-Holland, Amsterdam, 1977. MR 58:29439

[21] Xu Cheng-long and Guo Ben-yu, Laguerre pseudospectral method for nonlinear partial differential equations, J. Comp. Math., 20 (2002), 413-428.

[22] Xu Cheng-long and Guo Ben-yu, Mixed Laguerre-Legendre spectral method for incompressible fluid flow in an infinite strip, Advances in Comp. Math., 16(2002), 77-96.

Shanghai E-Institute for Scientific Computation, Department of Mathematics, Shanghai Normal University, Shanghai, 200234, Peoples Republic of China

Department of Applied Mathematics, Tongui University, Shanghai, 200092, Peoples Republic of China 